Review

\title{
Carrier-Envelope Offset Stabilized Ultrafast Diode-Pumped Solid-State Lasers
}

\author{
Stéphane Schilt * and Thomas Südmeyer \\ Laboratoire Temps-Fréquence, Université de Neuchâtel, Neuchâtel CH-2000, Switzerland; \\ E-Mail: Thomas.sudmeyer@unine.ch \\ * Author to whom correspondence should be addressed; E-Mail: stephane.schilt@unine.ch; \\ Tel.: +41-32-718-2917; Fax: +41-32-718-2901.
}

Academic Editor: Malte C. Kaluza

Received: 24 August 2015 / Accepted: 10 September 2015 / Published: 14 October 2015

\begin{abstract}
Optical frequency combs have been revolutionizing many research areas and are finding a growing number of real-world applications. While initially dominated by Ti:Sapphire and fiber lasers, optical frequency combs from modelocked diode-pumped solid-state lasers (DPSSLs) have become an attractive alternative with state-of-the-art performance. In this article, we review the main achievements in ultrafast DPSSLs for frequency combs. We present the current status of carrier-envelope offset (CEO) frequency-stabilized DPSSLs based on various approaches and operating in different wavelength regimes. Feedback to the pump current provides a reliable scheme for frequency comb CEO stabilization, but also other methods with faster feedback not limited by the lifetime of the gain material have been applied. Pumping DPSSLs with high power multi-transverse-mode diodes enabled a new class of high power oscillators and gigahertz repetition rate lasers, which were initially not believed to be suitable for CEO stabilization due to the pump noise. However, this challenge has been overcome, and recently both high power and gigahertz DPSSL combs have been demonstrated. Thin disk lasers have demonstrated the highest pulse energy and average power emitted from any ultrafast oscillator and present a high potential for the future generation of stabilized frequency combs with hundreds of watts average output power.
\end{abstract}

Keywords: optical frequency comb; carrier-envelope offset stabilization; modelocked laser; diode-pumped solid-state laser 


\section{Introduction}

Optical frequency combs produced by modelocked lasers have revolutionized many research areas over the last decade in the fields of optical metrology, spectroscopy or attosecond science, to name a few. Frequency combs provide a direct and phase-coherent link between optical and microwave frequencies, enabling the measurement of optical frequencies with extreme precision [1,2]. They constitute a key element of novel optical atomic clocks that demonstrated unprecedented frequency stability [3,4] and act as an optical-to-microwave frequency divider in ultra-low noise microwave oscillators [5]. A frequency comb is made of a huge number of optical modes of equal spacing and precisely known frequencies that are mutually phase-coherent. It thus constitutes a versatile tool for high-precision broadband spectroscopy [6,7].

Modelocked lasers have been produced from a variety of gain and host materials, including crystals, bulk glasses or fibers, to list the most widespread systems. However, only a few of them are classified as frequency combs. A frequency comb is a particular modelocked laser for which all independent parameters that define the optical spectrum are measured and/or controlled [8]. These parameters are the mode number $N$, the repetition rate frequency $f_{\text {rep }}$, i.e., the spacing between the modes, and the so-called carrier-envelope offset (CEO) frequency $f_{\text {CEO }}$ that represents the overall frequency-shift of the spectrum from the frequency origin. Therefore, each comb mode of frequency $v_{N}$ can be expressed by a simple relation as a function of these three parameters according to the well-known comb Equation [1]:

$$
v_{N}=N \cdot f_{\text {rep }}+f_{\text {CEO }}
$$

The repetition rate frequency $f_{\text {rep }}$ can be easily measured by detecting the optical pulse train at the output of the modelocked laser using a fast photodiode, and different means enable determining the mode number $N$ [9]. Therefore, the main challenge to make a frequency comb from a modelocked laser consists of detecting and stabilizing the CEO frequency $f_{\text {CEO. This was a significant contribution }}$ to the 2005 Nobel Prize in Physics awarded to T.W. Hänsch and J.L. Hall for the first fully-stabilized frequency combs $[1,10]$. The CEO frequency is traditionally detected using a nonlinear $f$-to- $2 f$ interferometry method [11], where the long wavelength part of an octave-spanning spectrum is frequency-doubled and heterodyned with the existing short wavelength comb components.

The first frequency combs were demonstrated from solid-state Ti:Sapphire lasers [11-13] as a result of their high peak power that is necessary to generate the coherent octave-spanning spectrum required for CEO detection in a nonlinear optical fiber. Other comb technologies have been developed shortly after, which present several advantages in terms of simplicity or reliable daily operation. Fiber lasers are nowadays the most largely spread comb systems, especially Er:fiber lasers emitting in the important $1.55 \mu \mathrm{m}$ window of the optical fiber telecommunications. Er:fiber lasers constitute a versatile tool for applications in ultrafast spectroscopy, confocal microscopy and precision metrology. For instance, they are widely used in conjunction to the dissemination of ultra-stable optical signals over large distances through the optical fiber network, which is the most efficient way today for distant comparison between optical atomic clocks [14]. But other types of fiber lasers have demonstrated frequency comb operation, such as Yb:fiber lasers in the $1 \mu \mathrm{m}$ spectral range [15] or Tm:fiber lasers at $2 \mu \mathrm{m}[16]$. Fiber lasers can generally be directly pumped by laser diodes, such as $980 \mathrm{~nm} \mathrm{AlGaAs}$ 
telecom pump diodes for Er:fiber and Yb:fiber lasers, or $1.56 \mu \mathrm{m}$ InGaAsP diodes for Tm:fiber lasers, which leads to compact and robust frequency comb systems. However, fiber lasers operate with high gain and high nonlinearities, which usually leads to higher noise, in particular a higher frequency noise of the CEO beat.

Besides the two mature technologies of Ti:Sapphire and fiber lasers, diode-pumped solid-state lasers (DPSSLs) have proved during the last years to constitute a promising alternative for the realization of frequency combs with state-of-the-art performance. DPSSLs combine several distinct advantages of the two aforementioned comb technologies. Like Ti:Sapphire lasers, they can emit a high average power directly from the oscillator, without external amplification. They also have low cavity losses (thus a high Q-factor) that lead to low intrinsic noise. This generally results in a lower noise of the free-running CEO beat that makes its tight locking to an external frequency reference less demanding in terms of feedback bandwidth. Finally, operation at high repetition rates is much less challenging than for fiber lasers as very compact cavities can be built. As a result, gigahertz or multi-gigahertz repetition rates can be achieved [17-21], which is highly attractive for applications in microwave photonics [22] or for the calibration of astronomical spectrographs [23]. Like fiber lasers, DPSSLs are directly pumped by laser diodes, which circumvents several disadvantages associated with the complexity, high cost and inefficient pumping of Ti:Sapphire combs. Pump diodes are very compact, efficient and low-cost, which makes DPSSLs very reliable and robust laser sources. Furthermore, pump diodes can be directly modulated via their injection current with a high bandwidth,

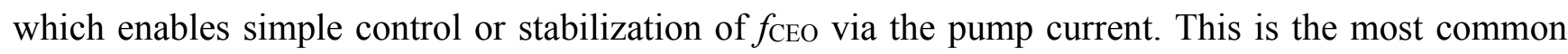
method to CEO-stabilize fiber laser frequency combs using the self-referencing $f$-to- $2 f$ interferometry method [24]. This method is applicable in a similar way in DPSSLs and constitutes the most common means to CEO-stabilize this type of femtosecond lasers [21,25-31].

In this review article, we present the current status of CEO-stabilized DPSSLs including some of our own achievements as well as works from other groups worldwide. We will restrict our review to DPSSL systems that have been CEO-stabilized or which are self-referenceable (i.e., for which a CEO beat has been detected by $f$-to- $2 f$ interferometry, but whose stabilization has not yet been realized), as the focus here is in their operation as frequency combs. An alternative scheme to stabilize the comb offset frequency consists of locking one comb mode to an optical frequency standard, e.g., a continuous wave laser referenced to some molecular or atomic transition. This is for instance a natural approach in Kerr-combs generated by parametric frequency conversion in continuously-pumped whispering gallery mode micro-resonators [32]. As the pump frequency coincides in this case with one line of the comb spectrum, it directly controls the offset frequency [33]. Such a scheme that circumvents the need for a coherent octave-spanning spectrum has also been applied in a few cases with fiber modelocked lasers [34,35], but we are not aware of any implementation with a DPSSL comb. Furthermore, this approach generally leads to less accurate comb frequencies. Therefore, phase-stabilization of $f_{\text {CEO }}$ to a stable radio-frequency (RF) signal such as provided by a GPS-disciplined atomic clock using $f$-to- $2 f$ interferometry leads to an absolute frequency grid that is required in many practical applications. For all these reasons, this review addresses only DPSSL combs that make use of $f$-to- $2 f$ interferometry. We will consider both DPSSLs made of a bulk gain material and thin disk lasers (TDLs) that offer high power scaling capabilities. TDLs demonstrated record high multi-100 $\mathrm{W}$ average power [36] that makes them very attractive for high field physics 
experiments such as high-harmonic generation (HHG) [37]. TDLs incorporate a thin (typically 50-250 $\mu \mathrm{m}$ ) disk-shaped gain material used in reflection in the laser cavity and pumped by high-power fiber-coupled diodes in a multi-pass configuration that enables a high pump absorption efficiency to be achieved despite the low single-pass absorption of the disk.

Beside DPSSLs based on bulk gain media and TDLs, ultrafast dielectric waveguide lasers constitute another promising technology for future compact frequency comb generation with laser diodes pumping. These lasers combine advantages of ion-doped solid-state and semiconductor lasers, with the potential to achieve high output powers with excellent beam quality and wafer-scale production capability with a high level of integration. However, these high power waveguides have hardly been modelocked so far and have thus not demonstrated any frequency comb operation yet. Therefore, such lasers will not be considered in this review.

The content of this paper is organized as follows. In Section 2, we review the different requirements and steps involved in the CEO-stabilization of a frequency comb by self-referencing, which covers nonlinear interferometry for CEO detection, nonlinear spectral broadening of the modelocked laser to a coherent octave-spanning spectrum, and some important aspects of a CEO stabilization loop. In Section 3, we review the main achievements on frequency combs produced by DPSSLs based on a bulk gain material, and in Section 4 for diode-pumped TDLs. Finally, a conclusion is presented in Section 5.

\section{Optical Frequency Comb Self-Referencing}

Strictly speaking, the self-referencing of a frequency comb originally referred to the detection of

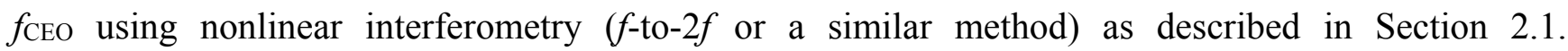
By extension, self-referencing is often considered as the subsequent step consisting of stabilizing the CEO beat obtained in this way to an RF reference. We consider this situation throughout this paper when talking about comb self-referencing or self-referenced comb. In contrast, we denote as self-referenceable a frequency comb for which $f_{\text {CEO }}$ has been detected by $f$-to- $2 f$ interferometry but has not yet been stabilized. We review here the principal steps and requirements to achieve comb self-referencing.

\subsection{Nonlinear Interferometry for $f_{C E O}$ Detection}

The physical origin of $f_{\mathrm{CEO}}$ arises from the difference between group and phase velocities inside the laser resonator, which induces a slippage $\Delta \phi_{\mathrm{CEO}}$ between the carrier phase and the pulse envelope from one pulse to the next in the time domain (see Figure 1a). From the properties of the Fourier transform that links the time and frequency domains, this corresponds to a frequency shift $f_{\text {CEO }}$ in the spectral domain (Figure 1b):

$$
f_{\mathrm{CEO}}=\frac{\Delta \phi_{\mathrm{CEO}}}{2 \pi} f_{\mathrm{CEO}}
$$

The carrier-envelope offset frequency $f_{\text {CEO }}$ constitutes a global frequency shift of the comb spectrum, such that the comb lines are not exact harmonics of the repetition rate $f_{\text {rep. This is described }}$ by the previous Equation 1, where $f_{\text {CEO }}$ is located by definition in the frequency interval 
$0 \leq f_{\text {CEO }} \leq f_{\text {rep }} / 2$. The CEO frequency is not part of the comb optical spectrum and thus cannot be directly observed. The standard method to detect it was initially proposed by Telle et al. [11], and applied later for the first time by Jones et al. [13] and Apolonski et al. [38]. It makes use of nonlinear interferometry to perform a heterodyne beat between two harmonic spectral ranges of a comb spectrum. This requires a broad enough comb spectrum such that the two harmonic components end up in the same wavelength range for the heterodyne beat to be detected. The most common method uses $f$-to- $2 f$ interferometry [11], in which a red portion of the comb spectrum is frequency-doubled and heterodyned with the existing blue comb components as displayed in Figure 2, which requires the comb spectrum to cover at least one frequency octave (i.e., ranging from frequency $f$ to frequency $2 f$ ). Alternatively, nonlinear interferometry involving higher-order nonlinearities can be used with a narrower spectrum, such as $2 f$-to- $3 f$ that requires a spectrum covering only $2 / 3$ of an octave, but two nonlinear processes are needed to produce second and third harmonic components $[11,39]$.

(a)
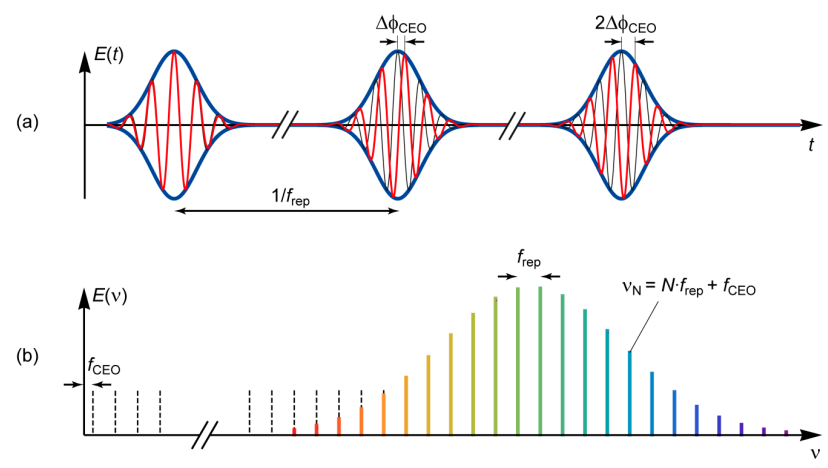

Figure 1. (a) Time domain pulse train emitted by a modelocked laser with the slippage $\Delta \phi_{\mathrm{CEO}}$ occurring from one pulse to the next between the carrier phase (shown by the thick red line) and the pulse envelope (blue line). (b) Spectral representation of a frequency comb in the frequency domain. The carrier-envelope offset frequency $f_{\text {CEO directly results }}$ from the carrier-envelope phase slippage $\Delta \phi_{\mathrm{CEO}}$ of the time domain from the properties of the Fourier transform that links the time and frequency representations.

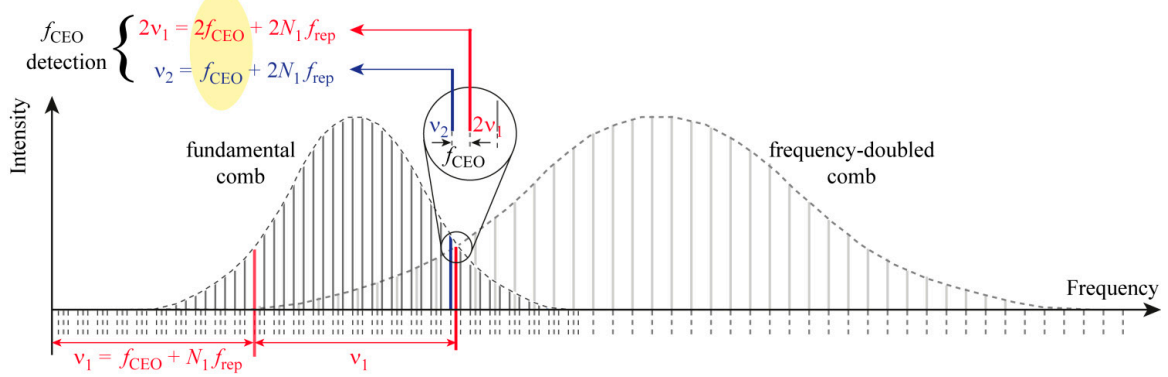

Figure 2. Principle of the $f$-to- $2 f$ interferometry method used to generate the CEO beat from an octave-spanning comb spectrum. A red component of the comb spectrum at $v_{1}=f_{\text {CEO }}+N_{1} \cdot f_{\text {rep }}$ is frequency-doubled and heterodyned with a blue component at $v_{2}=f_{\text {CEO }}+2 N_{1} \cdot f_{\text {rep }}$ to produce a beat at frequency $f_{\text {CEO }}$. In practice, the CEO beat does not result from the beat between a single pair of lines, but two finite spectral ranges (within the bandwidth of the frequency-doubling process) contribute and the CEO beat thus arises from the superimposition of beat signals between a multitude of lines pairs. 


\subsection{Coherent Supercontinuum Spectrum Generation}

External spectral broadening at the output of the modelocked laser is needed to achieve the broad spectrum that is required for CEO detection by nonlinear interferometry. The only laser system that is able to directly generate an octave-spanning spectrum in some circumstances is Ti:Sapphire as a result of its extremely broad gain bandwidth and high intra-cavity nonlinearities [40]. In addition, the coherence between the two spectral parts involved in the heterodyne beat must be preserved to enable a CEO beat with a sufficient signal-to-noise ratio (SNR) to be generated. The spectral broadening is obtained by supercontinuum (SC) generation in a nonlinear medium, such as highly nonlinear fiber (HNLF) or a photonic crystal fiber (PCF). SC generation relies on different physical mechanisms such as dispersion and nonlinear effects like self- or cross-phase modulation, Raman scattering, four wave mixing, and self-steepening to produce new frequency components in the comb spectrum while ideally preserving the coherence of the spectrum.

Spectral broadening in nonlinear fibers used in the anomalous dispersion regime (i.e., with a negative group delay dispersion coefficient $\beta_{2}$ ) or close to the zero-dispersion wavelength is particularly efficient as short and intense pulses can be maintained over a long propagation distance. In the presence of negative group velocity dispersion (GVD) and self-phase modulation (SPM) only, soliton propagation can be achieved in an optical fiber, in which GVD and SPM perfectly balance each other leading to a stationary pulse propagating along the fiber as a fundamental soliton. Higher-order solitons can also propagate in a quasi-stationary regime, where the pulse undergoes a periodic change with strong spectral and temporal effects, but retrieves its initial shape after a soliton period $z_{\text {sol }}=(\pi / 2) L_{D}$. This length depends on the magnitude of GVD through the dispersive length $L_{D}=T_{0}{ }^{2} /\left|\beta_{2}\right|$ and on the pulse duration $T_{0}$ [41]. However, in presence of other nonlinearities and higher order dispersion terms that are always present in optical fibers, a $N$ th order soliton splits into $N$ fundamental solitons after a fiber length of about $L_{\text {fiss }}=L_{D} / N$ [41]. This phenomenon is referred to as soliton fission. The resulting fundamental solitons then propagate as distinct and independent pulses with their own group velocity and are red-shifted by Raman scattering. In the normal dispersion regime at wavelengths below the zero-dispersion, dispersive waves are produced from a resonant energy transfer from the solitons, which extends the spectrum towards short wavelengths. The length of the nonlinear fiber is generally optimized such that the intense Raman soliton at long wavelengths and the dispersive wave at short wavelengths are separated by exactly one frequency octave. Therefore these spectral components efficiently contribute to the CEO beat generated in an $f$-to- $2 f$ interferometer. An example of SC spectrum generated in a PCF from the output of a $\mathrm{GHz} \mathrm{Yb}$ :CALGO $\left(\mathrm{CaGdAlO}_{4}\right)$ DPSSL is shown in Figure 3. Numerical simulations of the SC spectrum can be performed using a split-step Fourier-transform algorithm [41] that takes into account parameters such as the dispersion curve and nonlinear coefficients of the fiber and the properties of the pulses launched into the fiber. The results are generally in a good agreement with the experimentally observed SC spectrum when the fiber parameters are known with a sufficient precision (see Figure 3).

The soliton order $N_{\text {sol }}$ of the initial pulse depends on the ratio between the dispersive length $L_{D}$ and the nonlinear length $L_{N L}=1 /\left(\gamma P_{0}\right)$, where $P_{0}$ is the pulse peak power and $\gamma$ the nonlinear coefficient:

$$
N_{\text {sol }}=\sqrt{L_{D} / L_{N L}}
$$




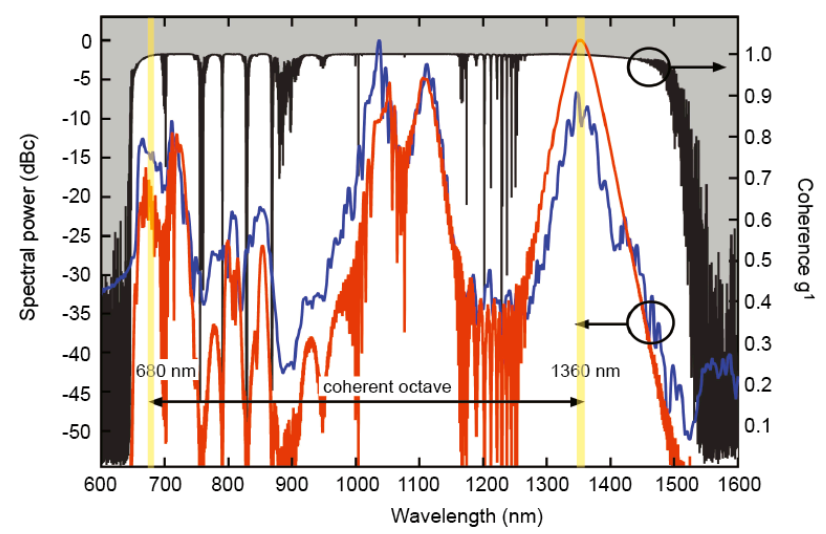

Figure 3. Octave-spanning SC spectrum generated in a PCF from the output of an Yb:CALGO DPSSL with gigahertz repetition rate: comparison between the measured (blue) and simulated (red) spectra. The Raman soliton at $1360 \mathrm{~nm}$ and the dispersive wave at $680 \mathrm{~nm}$ (shown by the yellow areas) are distant of one octave and have a high degree of coherence $g^{1}$ shown by the black curve displayed with respect to the right vertical axis; they are used to generate the CEO beat in an $f$-to- $2 f$ interferometer. (Reproduced with permission from [21]. (C) The Optical Society of America, 2014).

The soliton order $N_{\text {sol }}$ constitutes a useful metric to estimate the degree of coherence of the generated SC spectrum. It was shown that a coherent SC spectrum requires a soliton order $N_{\text {sol }}<10$ at the input of the nonlinear fiber [19,41]. An incoherent regime is achieved when $N_{\text {sol }}>10$ as a result of modulation instabilities that amplify small noise at the fiber input to cause large deviation of the intensity, timing, and phase of the output SC spectrum. Therefore, SC generation for frequency comb self-referencing always requires a soliton order $N_{\text {sol }}<10$.

Besides the use of HNLFs and PCFs, an integrated silicon-nitride ( $\left.\mathrm{Si}_{3} \mathrm{~N}_{4}\right)$ waveguide embedded in $\mathrm{SiO}_{2}$ recently proved to constitute a powerful nonlinear medium for coherent SC generation for frequency combs [42]. Using $\mathrm{Yb}$ femtosecond DPSSLs in the $1 \mu \mathrm{m}$ spectral range with $100 \mathrm{MHz}$ and $1 \mathrm{GHz}$ repetition rates, Meyer et al., obtained a coherent SC spectrum enabling CEO detection by $f$-to- $2 f$ interferometry with $>25 \mathrm{~dB}$ SNR for the $100 \mathrm{MHz}$ laser (and $>30 \mathrm{~dB}$ for the $1 \mathrm{GHz}$ laser). These results were achieved with a peak power lowered by more than a factor 10 compared to the use of a commercial PCF and in a length shorter than $10 \mathrm{~mm}$ (compared to typically $1 \mathrm{~m}$ for the PCF), as a result of the high nonlinear refractive index of $\mathrm{Si}_{3} \mathrm{~N}_{4}$ that is 10 times larger than for silica.

\subsection{CEO Stabilization Loop}

The detection of a CEO beat with a sufficient SNR by $f$-to- $2 f$ interferometry after SC generation constitutes a first mandatory step for the self-referencing stabilization of a frequency comb. A modelocked laser is sometimes said to be self-referenceable once this CEO detection has been achieved. However, the subsequent stabilization of $f_{\text {CEO }}$ is not a straightforward step, especially to achieve a tight CEO lock. The most common method for CEO stabilization consists of phase-locking $f_{\text {CEO }}$ to an external reference oscillator, or to a sub-harmonic of the repetition rate $f_{\text {rep }}$ in some cases, using a phase-locked loop (PLL). A first requirement for a proper operation of such a PLL is to have a sufficient SNR of the CEO beat. A rule-of-thumb typically requires a SNR larger than $\sim 25 \mathrm{~dB}$. 
A PLL is typically made of three distinct components (Figure 4): (i) a phase detector that measures the phase fluctuations between $f_{\text {CEO }}$ and the reference signal to generate an error signal; (ii) a proportional-integral-derivative (PID) servo-controller that amplifies the error signal to produce

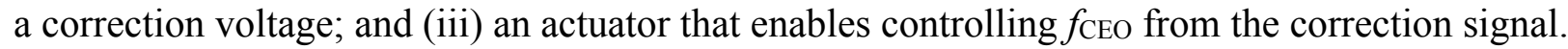

A large phase noise generally affects the CEO beat, whose free-running linewidth is typically in the range from a few kilohertz [27] to a few megahertz [43]. Consequently, a double-balanced mixer (DBM) cannot be directly used as a phase detector as a result of its narrow operation range. The CEO beat is thus either frequency-divided before being compared to a reference signal in a DBM, or a digital phase detector with a much larger linear phase response is used to measure the phase fluctuations of the CEO beat. For instance, in our comb CEO stabilization experiments $[21,27,28,44]$, we typically used the digital phase detector DXD200 [45] that is also implemented in the frequency combs of the market leader MenloSystems (Martinsried/Munich, Germany) and provides a large linear phase response of $\pm 64 \pi$.

The availability of a suitable actuator to control $f_{\text {CEO }}$ is another key component for the successful implementation of a PLL to phase-stabilize the CEO beat. The actuator should provide a linear control of $f_{\text {CEO }}$ over a sufficiently large range to keep a stable long-term lock. The bandwidth of the actuator, i.e., the speed at which it can act on $f_{\text {CEO }}$ to correct for fast fluctuations, is another key parameter to achieve a proper stabilization. A broad linewidth of the free-running CEO beat mainly arises from high frequency noise components. Therefore, a fast actuator is required to correct for these components if a significant linewidth reduction is aimed for, for instance to achieve a tight phase lock. A tight lock is characterized by the suppression of the full width at half maximum (FWHM) linewidth of the signal and the appearance of a coherent peak (delta function) in the center of the RF spectrum, surrounded by a noise pedestal that corresponds to the residual phase noise of the signal (Figure 5b). A tight lock is typically achieved when the integrated phase noise $\Delta \phi_{r m s}=\left[\int S_{\phi}(f) d f\right]^{1 / 2}$ is smaller than 1 [46], where $S_{\phi}(f)$ is the phase noise power spectral density (PSD). In such a case, a fraction $\eta=\exp \left[-\int S_{\phi}(f) d f\right]$ of the signal power is in the coherent peak. When a tight lock cannot be achieved, the stabilized signal keeps a finite linewidth and one generally refers to this situation as a loose lock (Figure 5a).

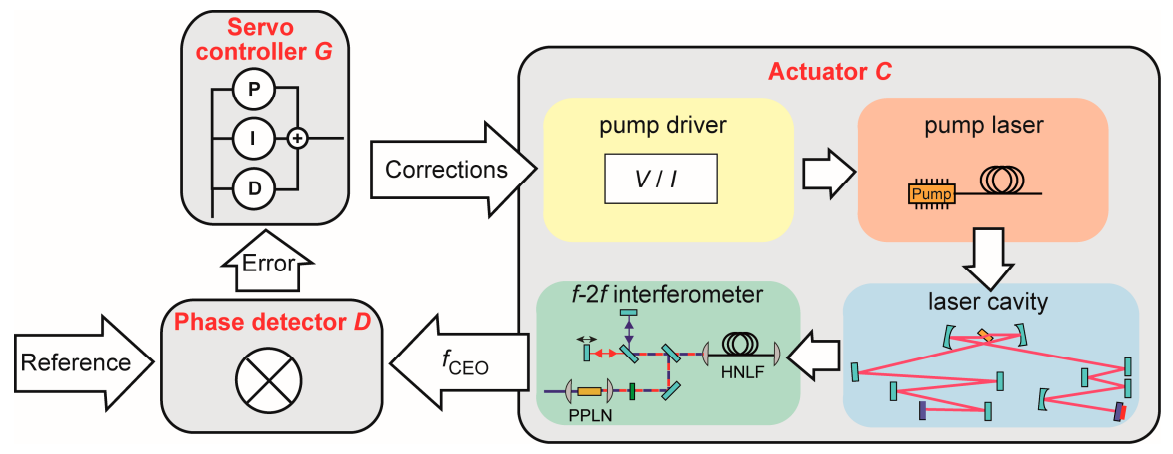

Figure 4. Scheme of principle of a PLL for coherent stabilization of $f_{\mathrm{CEO}}$ to an external reference frequency, with its three main components: (i) a phase detector $D$ to measure the phase fluctuation between the signal and the reference to produce an error signal; (ii) a PID servo-controller $G$ that amplifies the error signal; and (iii) an actuator $C$ that tunes $f_{\mathrm{CEO}}$ from a control voltage. 
(a)
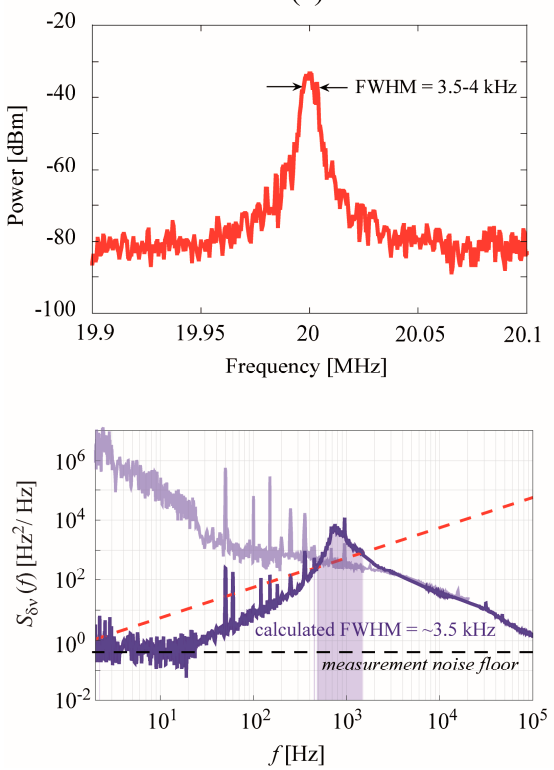

(b)
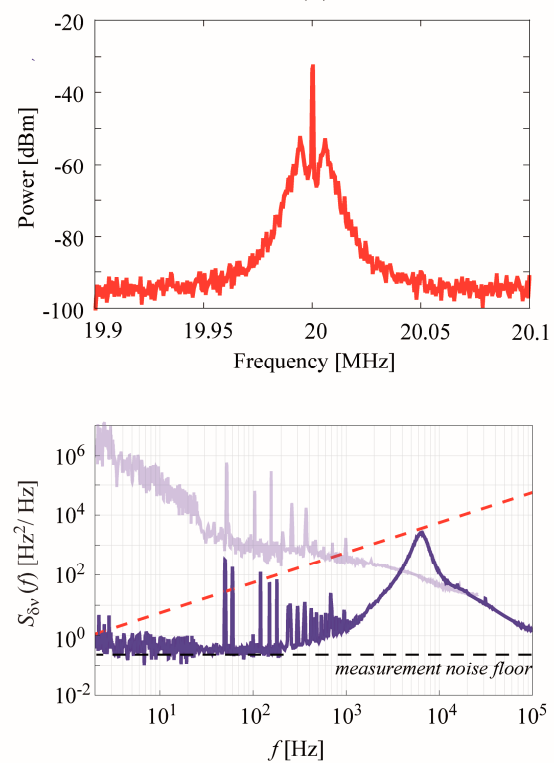

Figure 5. (a) Loose lock and (b) tight lock achieved for the CEO beat in an Er:Yb:glass DPSSL comb $[27,28]$. The upper graphs show the RF spectrum with the presence of a coherent carrier in the case of the tight lock. The lower graphs show the corresponding frequency noise PSD for the free-running (grey curves) and stabilized (blue curves) CEO beat. The linewidth of the locked CEO beat can be approximated using the $\beta$-separation line introduced by Di Domenico et al. [47] (dashed red line). In the case of a loose lock (a), a finite linewidth is obtained that is proportional to the colored area for which the frequency noise PSD exceeds the $\beta$-separation line. For a tight lock (b), the frequency noise PSD is completely reduced below the $\beta$-separation line, which corresponds to a FWHM linewidth reduced to zero that is representative of the presence of a coherent carrier.

A simple approximation to determine the linewidth of a laser or of a beat signal from an arbitrary frequency noise spectrum was proposed by Di Domenico et al., based on the concept of the $\beta$-separation line [47]. This line shown in Figure 5 and defined as $S_{\delta v}(f)=\left(8 \ln (2) / \pi^{2}\right) \cdot f$ splits the frequency noise PSD $S_{\delta v}(f)$ into two areas with a strongly different impact on the lineshape. Only the noise components for which $S_{\delta v}(f)>\left(8 \ln (2) / \pi^{2}\right) \cdot f$, corresponding to the slow modulation area, contribute to the FWHM linewidth of the signal. The other components for which $S_{\delta v}(f)<\left(8 \ln (2) / \pi^{2}\right) \cdot f$ (fast modulation area) only affect the wings of the lineshape, without contributing to the FWHM linewidth. A simple approximation of the FWHM linewidth is obtained from the surface $A$ of the slow modulation area corresponding to the geometrical area under the frequency noise PSD obtained for all Fourier frequencies for which $S_{\delta v}(f)$ exceeds the $\beta$-separation line [47]:

$$
\begin{gathered}
F W H M=\sqrt{8 \ln (2) A} \\
A=\int H\left(S_{\delta v}(f)-8 \ln (2) f / \pi^{2}\right) S_{\delta v}(f) d f
\end{gathered}
$$

with $H(x)$ being the Heaviside step unit function $(H(x)=1$ if $x \geq 0$ and $H(x)=0$ if $x<0)$. An experimental validation of the approximated linewidth given by Equation (5) performed with CEO 
beat signals of fiber and DPSSL frequency combs proved its accuracy (within the experimental uncertainties) over more than three decades of linewidth values [48]. The concept of the $\beta$-separation line offers a simple tool to evaluate the feedback bandwidth that is required to achieve a tight lock in a stabilization loop [47]. As a tight lock is characterized by the suppression of the signal linewidth, the stabilization loop must be able to reduce all noise components that contribute to the linewidth below the $\beta$-separation line. The highest frequency contributing to the signal linewidth corresponds to the crossing point between the frequency noise PSD and the $\beta$-separation line. Therefore, this crossing point constitutes a useful metric for the required loop bandwidth.

The most commonly used actuator to tune and stabilize $f_{\mathrm{CEO}}$ in DPSSLs and fiber lasers is the current of the pump diode. The pump current has a direct influence on the pulse parameters of the femtosecond laser, such as pulse duration and energy, which directly translates into a change of $f_{\text {CEO. }}$ CEO stabilization by pump current modulation has thus been implemented in various DPSSLs that will be described in more details in Sections (3) and (4) [21,25-31]. However, the pump current acts onto $f_{\text {CEO }}$ through the gain medium, and the corresponding modulation bandwidth is limited by the upper-state lifetime of the gain material that induces a roll-off in the modulation response of $f_{\text {CEO. The }}$ upper-state lifetime is in the millisecond range for many important diode-pumped $\mathrm{Yb}$ and $\mathrm{Er}$ gain materials, which limits the CEO modulation bandwidth to the kilohertz or tens of kilohertz range $[21,26,27]$. Extending the feedback bandwidth beyond the limit of the gain upper-state lifetime can be achieved using a phase-lead filter in the loop [26], or by the use of a derivative component in the servo-controller. However, other approaches to circumvent the gain lifetime limitation and to act on the CEO beat frequency at a higher speed have been proposed based on the use of intra-cavity loss-modulation. As an example, a graphene electro-optic modulator was used in reflection to stabilize the CEO frequency in a Tm-fiber oscillator with a large feedback loop bandwidth of $>1 \mathrm{MHz}$ [16]. Another method to overcome the gain lifetime limitation is opto-optical modulation (OOM) of a semiconductor device inside the cavity. Using a SESAM as a fast actuator for CEO frequency control in a modelocked DPSSL resulted in a feedback loop bandwidth $>100 \mathrm{kHz}$ [44]. The results will be described in more details in Section 3.3.

A high SNR of the free-running CEO beat with a low enough frequency noise are prerequisites to achieve the self-referencing of an optical frequency comb as previously discussed in this section. However, these conditions may not be sufficient in some particular cases where the dynamics of $f_{\mathrm{CEO}}$ may play a crucial role. Such a case was shown and investigated by Bucalovic et al. in an Er:Yb:glass DPSSL where $f_{\text {CEO }}$ is controlled by the pump current [49] and another case will be discussed in Section 4 for a TDL. In the Er:Yb:glass laser, a reversal point occurred in the static response of $f_{\text {CEO as }}$ a function of the pump current $I_{\text {pump }}$, where the slope of $\partial f_{\text {CEO }} / \partial I_{\text {pump }}$ changed its sign (Figure 6a). A tight phase-lock of $f_{\text {CEO }}$ to an external frequency reference of $20 \mathrm{MHz}$ was straightforwardly achieved at pump currents located below the reversal point, whereas no stabilization at all (even a loose lock) could be achieved at pump currents located on the other side of the reversal point, despite a slightly higher SNR and a very similar frequency noise of the free-running CEO beat. The reason behind this effect was shown to be related to the dynamic response of $f_{\text {CEO }}$ to pump current

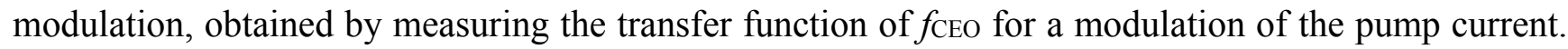
Not only the amplitude, but also the phase of the transfer function is of particular importance for the realization of a stable feedback loop. According to a general rule-of-the-thumb in control systems, a 
sufficient phase margin is needed at the unity gain frequency to keep a feedback loop stable [50]. As a consequence, the overall phase shift accumulated throughout the loop has to be kept sufficiently higher than $-180^{\circ}$ to prevent the system to turn into a positive feedback loop leading to an unstable operation. In the considered laser, the high frequency range of the transfer function (above $\sim 1 \mathrm{kHz}$ ) did not depend on the operating pump current (neither in amplitude nor in phase), whereas a significant difference occurred in the low frequency range. In particular, the phase showed a detrimental change of $180^{\circ}$ between low and high pump current (Figure 6c). This behavior indicated that two different

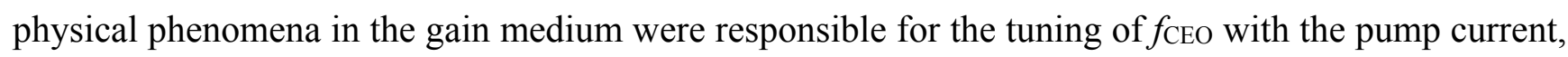
one of which being of thermal origin and responsible for the detrimentally different phase behavior observed on one side of the reversal point where the stabilization could not be achieved. This work demonstrated the importance of the CEO dynamics in the self-referencing of a DPSSL frequency comb.

(a)

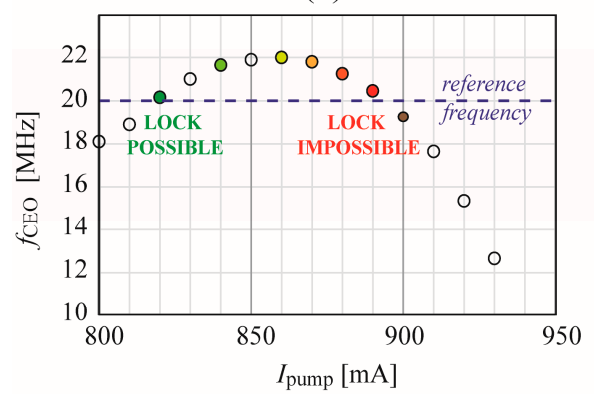

(b)

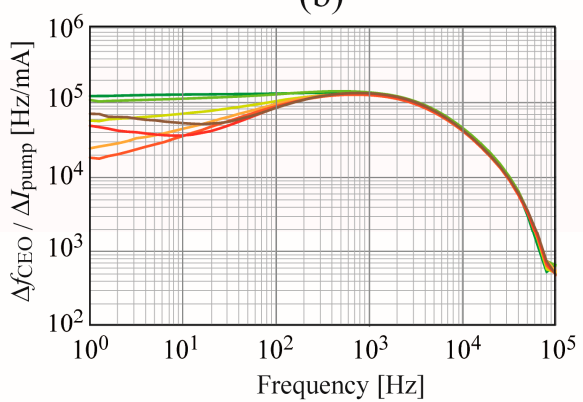

(c)

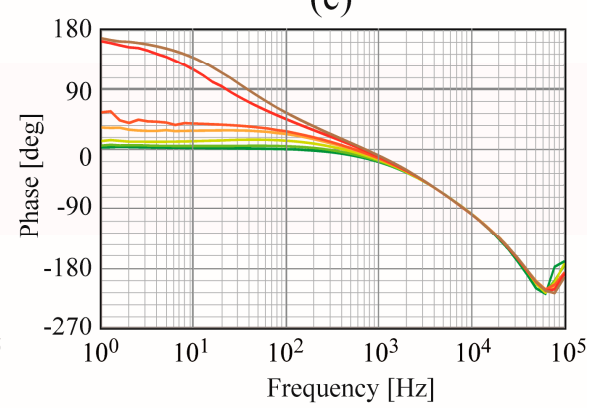

Figure 6. (a) Static tuning curve of $f_{\text {CEO }}$ with respect to the pump current showing a reversal point at $I_{\text {pump }} \approx 860 \mathrm{~mA}$. The CEO frequency is stabilized to $20 \mathrm{MHz}$ in this DPSSL comb, which can be realized in principle at two pump currents located on each side of the reversal point. (b) Amplitude and (c) phase of the dynamic response of $f_{\text {CEO }}$ to pump current modulation measured at different pump currents below the reversal point (green curves), at the reversal point (yellow curve) and above the reversal point (orange to red curves).

\subsection{CEO vs. CEP Stabilization}

The previously discussed frequency comb self-referencing where $f_{\text {CEO }}$ is phase-locked to an external RF reference signal consists of stabilizing the CEO frequency $f_{\text {CEO }}$. In such a case, the carrier slips by a constant phase difference $\Delta \phi_{\text {CEO }}$ relative to the pulse envelope between two consecutive pulses according to Equation (2), but this phase slip is not zero. As a consequence, the phase difference between the carrier and the envelope (the so-called carrier-envelope phase (CEP)) varies from pulse to pulse and consecutive pulses do not exactly reproduce themselves. An identical pulse is retrieved only after a certain number of pulses given by the ratio $f_{\text {rep }} / f_{\text {CEO }}$ (Figure $7 \mathrm{a}$ ). Most of the combs applications in frequency metrology or spectroscopy rely on such CEO-stabilized combs where the absolute phase of the carrier within the pulse is irrelevant. It is only in the particular case where $f_{\text {CEO }}=0$ that a constant CEP is achieved and successive pulses are identical (Figure 7b). In such a case, the comb is said to be CEP-stabilized. This condition is stronger than CEO-stabilized, as it requires a constant 
carrier phase to be achieved with respect to the pulse envelope in all pulses and not only a constant change of the phase difference between the carrier and pulse envelope from one pulse to the next. Therefore, a CEP-stabilized laser is always also CEO-stabilized, whereas the reverse is generally not true (excepted in the particular case of $f_{\mathrm{CEO}}=0$ ). However, even if all pulses are identical when $f_{\text {CEO }}=0$, their absolute CEP remains arbitrary and different CEP values lead to the same comb frequencies $v_{N}=N f_{\text {rep }}$.

(a)
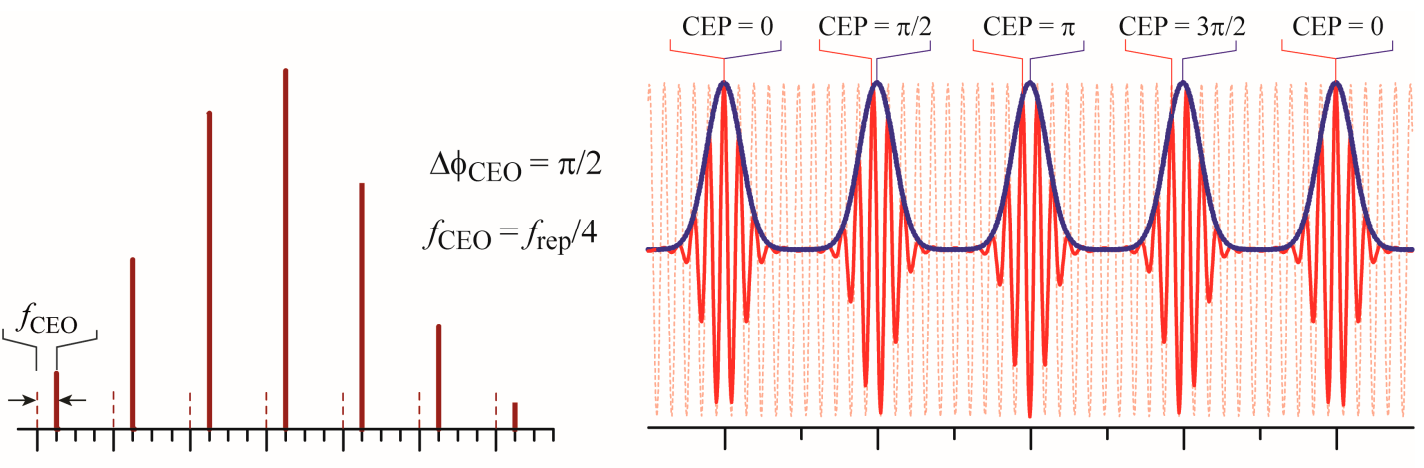

(b)
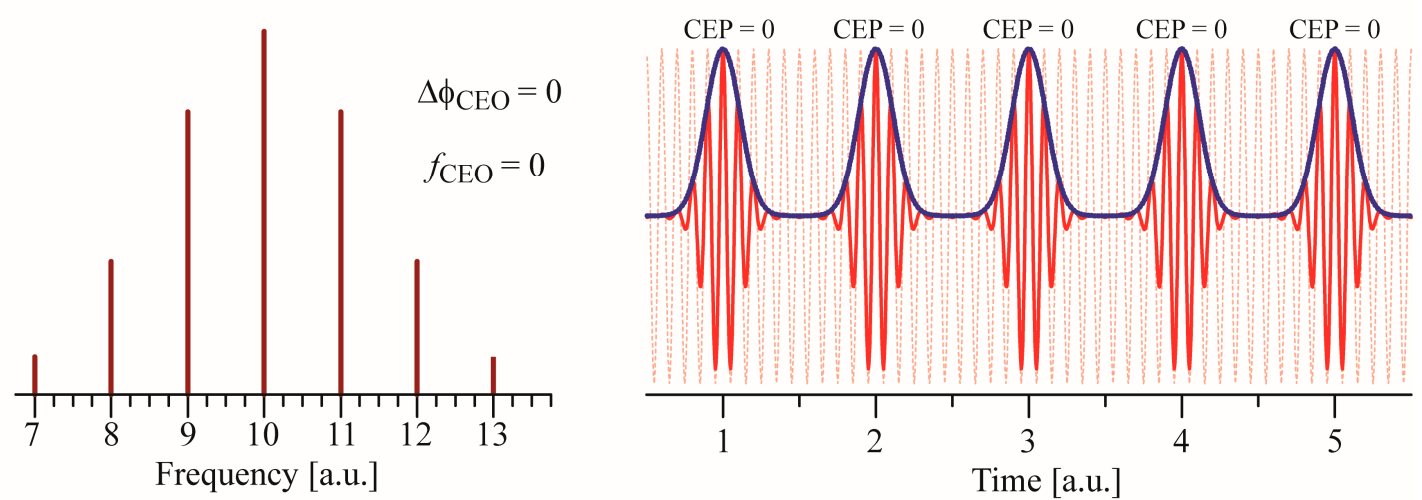

Figure 7. Illustrative examples of the correspondence between frequency (left) and time (right) representations of a pulse train emitted by a modelocked laser [51]. The red oscillation shows the evolution of the carrier and the blue line represents the pulse envelope. (a) $f_{\mathrm{CEO}}=f_{\text {rep }} / 4, \Delta \phi_{\mathrm{CEO}}=\pi / 2$ : an identical pulse is retrieved after four pulses; (b) $f_{\mathrm{CEO}}=0, \Delta \phi_{\mathrm{CEO}}=0$ : all pulses are identical (shown here for the particular case where $\mathrm{CEP}=0$, however, the absolute value of CEP can be arbitrary).

CEP stabilization is of particular importance in some time domain experiments where a physical effect under study occurs for a single pulse. For instance, extreme nonlinear optical experiments are directly sensitive to the electric field and not to the intensity of a light pulse. Therefore, they directly depend on the "absolute" CEP [51]. A typical example is high harmonic generation (HHG) where a gas target illuminated by an intense laser pulse emits high harmonics of the incident light frequency, which is a means to produce extreme ultra-violet frequency combs.

Currently it is possible to determine and stabilize the change of the carrier-envelope phase between successive pulses via the CEO frequency as previously described. However, extending this to the direct measurement of the carrier-envelope phase itself is much more challenging. Stabilizing the CEO frequency to $f_{\mathrm{CEO}}=0$ is a first step in this direction, as it enables the production of a train of identical pulses (zero carrier-envelope phase shift $\Delta \phi_{\mathrm{CEO}}$ ). However, the absolute CEP is still not controlled in 
this case. Self-referencing a frequency comb with $f_{\text {CEO }}=0$ cannot be achieved using the standard negative feedback loop approach used to lock $f_{\text {CEO }}$, but an alternative method based on a feedforward stabilization [52] makes it possible. A passive stabilization of $f_{\text {CEO }}$ to zero was also realized in an Er:fiber modelocked laser via difference frequency generation between two distant parts of a SC spectrum generated in an HNLF, namely a $2 \mu \mathrm{m}$ soliton and a dispersive wave at $860 \mathrm{~nm}$, to produce a broadband CEO-free output spectrum centered at the oscillator wavelength of $1.55 \mu \mathrm{m}$ [53].

\section{Self-Referenced DPSSLs with Bulk Gain Material}

Several frequency combs have been demonstrated from modelocked DPSSLs with bulk gain materials in the last decade. Most of them are based on the use of a semiconductor saturable absorber mirror (SESAM) as a nonlinear reflector in the resonator to favor the generation of ultrashort pulses [54]. SESAM-modelocking has the advantage of being self-starting, and generally leads to a reliable laser operation over extended periods without the need for significant optical realignment. The other principal modelocking mechanism that is used in femtosecond DPSSLs is Kerr-lens modelocking (KLM). KLM relies on an artificial saturable absorber resulting from nonlinearities in the gain medium that induces a Kerr lens. The Kerr lens focuses intense pulses on an aperture and thus reduces optical losses for ultrashort pulses. KLM enables ultrashort pulse durations, but is usually not self-starting.

In this section, we will review the main achievements realized in terms of CEO-stabilized DPSSLs. Table 1 lists the main parameters of these lasers.

\subsection{Chromium-Doped Lasers}

The first CEO detection from a DPSSL was demonstrated in 2001 by Holzwarth et al., in a Kerr-lens modelocked Cr:LiSAF laser [55]. This laser was pumped by two $670 \mathrm{~nm}$ diodes with a total power of $700 \mathrm{~mW}$ to produce $115 \mathrm{~mW}$ average output power at $894 \mathrm{~nm}$ in modelocked operation with $\sim 60$ fs pulse duration and $93 \mathrm{MHz}$ repetition rate. A CEO beat signal with an SNR exceeding $40 \mathrm{~dB}$ in a $100 \mathrm{kHz}$ resolution bandwidth (RBW) was obtained after spectral broadening in a PCF and an $f$-to- $2 f$ interferometer, but the CEO was not stabilized due to the limited access to the sealed box of the LiSAF laser as explained by the authors. The pump diodes were driven by a battery for low-noise operation.

A femtosecond laser based on a bulk Cr-doped material, namely Cr:forsterite, has been fully-stabilized and referenced to an H-maser [43]. However, this oscillator was not directly pumped by a laser diode, but by an $\mathrm{Yb}$ :fiber laser. Therefore, it is not exhaustively discussed here, but only briefly mentioned to highlight one of the key benefits of DPSSLs that is the direct pump current modulation capability for self-referencing. Instead, an acousto-optic modulator was needed in this laser system for pump power control, as direct pump modulation was not feasible. 
Table 1. List and main parameters of CEO-stabilized or self-referenceable DPSSLs reported in the literature (bulk DPSSLs and TDLs).

\begin{tabular}{|c|c|c|c|c|c|c|c|c|c|c|c|c|}
\hline Type & Gain & $\begin{array}{c}\lambda \\
(\mathbf{n m})\end{array}$ & $\begin{array}{l}P_{\text {av }} \\
(\mathbf{W})\end{array}$ & $\begin{array}{c}f_{\text {rep }} \\
(\mathbf{G H z})\end{array}$ & $\begin{array}{l}\Delta \tau \\
(\mathbf{f s})\end{array}$ & ML & Pump & $\begin{array}{c}\text { CEO } \\
\text { Control } \\
\end{array}$ & $\begin{array}{l}\text { CEO Lock } \\
\text { BW (kHz) }\end{array}$ & CEO Integr. Noise (mrad) & Other & Refs. \\
\hline \multirow{12}{*}{$\begin{array}{c}\text { Bulk } \\
\text { DPSSLs }\end{array}$} & Cr:LiSAF & 894 & 0.115 & 0.093 & $\sim 60$ & KLM & STM & \multicolumn{3}{|c|}{ not stabilized } & - & {$[55]$} \\
\hline & Yb:KYW & 1033 & 0.250 & 0.16 & $\sim 300$ & SESAM & FBG-STM & $I_{\text {pump }}$ & 100 & $300[0.1 \mathrm{~Hz}-1 \mathrm{MHz}]$ & $\begin{array}{c}\text { External amplification }+ \\
\text { compression }\end{array}$ & {$[25,26]$} \\
\hline & $\mathrm{Yb}: \mathrm{KGW}$ & 1042 & 2.2 & 1.0 & $\sim 290$ & SESAM & STM & \multicolumn{3}{|c|}{ not stabilized } & External compression & [19] \\
\hline & $\mathrm{Yb}: \mathrm{KGW}$ & 1047 & 3.4 & 1.06 & 125 & SESAM & MTM & \multicolumn{3}{|c|}{ not stabilized } & - & {$[20]$} \\
\hline & $\mathrm{Yb}: \mathrm{KGW}$ & 1040 & 0.6 & 0.133 & 100 & SESAM & MTM & $I_{\text {pump }}$ & $\sim 300$ & $407[1 \mathrm{~Hz}-5 \mathrm{MHz}]$ & - & {$[56]$} \\
\hline & Yb:CALGO & 1062 & 1.7 & 1.0 & $\sim 60$ & SESAM & MTM & $I_{\text {pump }}$ & $\sim 300$ & $744[1 \mathrm{~Hz}-5 \mathrm{MHz}]$ & - & {$[21]$} \\
\hline & Yb:CALGO & 1055 & 1.7 & 1.0 & 63 & SESAM & VHG-MTM & $I_{\text {pump }}$ & $<200$ & $339[1 \mathrm{~Hz}-5 \mathrm{MHz}]$ & - & {$[57]$} \\
\hline & $\mathrm{Yb}$ & 1047 & 0.205 & 0.1 & 111 & SESAM & FBG-STM & $I_{\text {pump }}$ & $\sim 130$ & $83[1 \mathrm{~Hz}-1 \mathrm{MHz}]$ & - & [29] \\
\hline & Yb:KGW & 1031 & 0.215 & 0.1 & 155 & SESAM & & $I_{\text {pump }}$ & $\sim 100$ & $1100[1 \mathrm{~Hz} ; 1 \mathrm{MHz}]$ & External compression & {$[58]$} \\
\hline & Er:Yb:glass & 1558 & 0.11 & 0.075 & 170 & SESAM & FBG-STM & $I_{\text {pump }}$ & 5.5 & $720[1 \mathrm{~Hz}-100 \mathrm{kHz}]$ & - & {$[27,28]$} \\
\hline & Er:Yb:glass & 1558 & 0.11 & 0.075 & 170 & SESAM & FBG-STM & OOM & $40-50$ & $65(30 *)[1 \mathrm{~Hz}-100 \mathrm{kHz}]$ & - & {$[44]$} \\
\hline & Er:Yb:glass & 1560 & 0.115 & 0.1 & 190 & SESAM & FBG-STM & AOFS-FB & not reported & 118 & - & {$[59]$} \\
\hline \multirow{5}{*}{$\begin{array}{c}\text { Thin } \\
\text { Disk } \\
\text { Lasers }\end{array}$} & $\mathrm{Yb}: \mathrm{Lu}_{2} \mathrm{O}_{3}$ & 1035 & 7 & 0.064 & 142 & SESAM & VHG-MTM & \multicolumn{3}{|c|}{ not stabilized } & - & {$[60]$} \\
\hline & Yb:YAG & 1030 & 17 & 0.038 & 250 & KLM & MTM & $\begin{array}{c}I_{\text {pump }} \\
\text { IC-AOM }\end{array}$ & not reported & $\begin{array}{c}250100 / 200(\mathrm{ool} * *) \\
{[1 \mathrm{~Hz}-500 \mathrm{kHz}]}\end{array}$ & External compression & {$[61]$} \\
\hline & Yb:YAG & 1030 & 40 & 0.038 & 250 & KLM & MTM & IC-AOM & not reported & $\begin{array}{l}180 / 270(\mathrm{ool} * *) \\
{[1 \mathrm{~Hz}-500 \mathrm{kHz}]}\end{array}$ & External compression & {$[62]$} \\
\hline & Yb:CALGO & 1052 & 2.1 & 0.065 & 70 & SESAM & VHG-MTM & $I_{\text {pump }}$ & $30-40$ & $120[1 \mathrm{~Hz}-1 \mathrm{MHz}]$ & - & {$[30]$} \\
\hline & $\mathrm{Yb}: Y A G$ & 1030 & 140 & 0.0095 & $\sim 950$ & SESAM & MTM & \multicolumn{3}{|c|}{ not stabilized } & External compression & {$[31,63]$} \\
\hline
\end{tabular}

ML: modelocking; STM: single-transverse-mode; MTM: multi-transverse-mode; FBG-STM: fiber Bragg-grating-stabilized single-transverse-mode; VHG-MTM: volume holographic grating-stabilized multi-transverse-mode; OOM: opto-optical modulation; AOFS-FB: acousto-optic frequency shifter feedback; IC-AOM: intra-cavity acousto-optic modulator. *Integrated phase noise without the contribution of technical noise at $50 \mathrm{~Hz}$ and harmonics; ** ool: out-of-loop measurement. 


\subsection{One-Micrometer Ytterbium-Doped Lasers}

Some years later, the first self-referenced Yb DPSSL in the $1 \mu \mathrm{m}$ spectral range was reported by Meyer et al. [25]. The Yb:KYW laser was pumped by two $600 \mathrm{~mW}$ fiber-coupled laser diodes at $980 \mathrm{~nm}$ stabilized with a fiber Bragg grating to produce $250 \mathrm{~mW}$ output power in $\sim 290 \mathrm{fs}$ at a repetition rate adjustable between $160 \mathrm{MHz}$ and $190 \mathrm{MHz}$. As a result of the quite long pulses emitted by this laser, coherent octave-spanning SC generation for CEO beat detection could not be directly achieved from the laser output, but required external amplification using an $\mathrm{Yb}$ :fiber amplifier and pulse compression. An average power of $300 \mathrm{~mW}$ compressed down to 113 fs was launched into a microstructured fiber to generate a coherent spectrum extending from $650 \mathrm{~nm}$ to $1450 \mathrm{~nm}$ that allowed $f_{\text {CEO }}$ to be detected in an $f$-to- $2 f$ interferometer with a SNR of $34 \mathrm{~dB}$ (in $100 \mathrm{kHz}$ RBW). The CEO frequency was stabilized by feedback to the current of one of the pump diodes with a loop bandwidth of about $20 \mathrm{kHz}$ limited by the roll-off response of $f_{\mathrm{CEO}}$ to the pump current modulation. In these conditions, an integrated phase noise of 1.7 radians [5 Hz-4 MHz] was obtained from the measured CEO frequency noise PSD [25]. Later on, improvements of the CEO lock were reported for the use of this frequency comb as an optical-to-microwave frequency divider for low-noise microwave generation [26]. With a total pump power of $830 \mathrm{~mW}$ and a repetition rate of $186 \mathrm{MHz}$, the CEO servo bandwidth was extended to $\sim 100 \mathrm{kHz}$ by the use of a phase-lead filter, which enabled overcoming the $\sim 45 \mathrm{kHz}$ limitation of the upper state lifetime of the $\mathrm{Yb}: \mathrm{KYW}$ crystal. In this condition, a residual integrated phase noise of $300 \mathrm{mrad}$ was achieved $[0.1 \mathrm{~Hz}-1 \mathrm{MHz}]$.

Frequency combs based on Yb DPSSLs in the $1 \mu \mathrm{m}$ range have become fairly popular in the last five years and have been the subject of several studies and developments. A research direction of particular interest has been the extension of combs operation to the range of gigahertz or multi-gigahertz repetition rate. High repetition rate lasers enable an easier access to the individual optical comb lines and an increased power per comb mode for a given average power that leads to an improved SNR in various applications. However, SESAM-modelocked DPSSLs have to overcome some challenges when increasing the repetition rate, as the decreased pulse energy may result in Q-switching instabilities [64].

A key achievement towards the self-referenced stabilization of gigahertz DPSSL combs was the first CEO detection realized by Pekarek and co-workers in an Yb:KGW laser [19]. Using $6 \mathrm{~W}$ of pump power from a distributed Bragg reflector (DBR) tapered laser diode at $980 \mathrm{~nm}$, more than $2 \mathrm{~W}$ of average power was emitted at $1042 \mathrm{~nm}$ in modelocked operation with a repetition rate of $1.0 \mathrm{GHz}$ and a pulse duration of $\sim 290$ fs based on SESAM-modelocking. The high output power of this oscillator enabled an octave-spanning SC to be generated in a PCF directly from the oscillator output, without any external amplification, which kept the system simple. However, the coherence of the generated SC spectrum was too low to detect a CEO beat, especially at the wavelengths used in the $f$-to- $2 f$ interferometer. This was confirmed by numerical simulations that showed that a soliton order of around 13 was achieved for the pulses launched into a PCF from this oscillator. By reducing the pulse duration to around $100 \mathrm{fs}$ using a passive fiber-compressor and using a lower average power of $1.1 \mathrm{~W}$, the soliton order was reduced to five, enabling the detection of a CEO beat at $680 \mathrm{~nm}$ with an SNR of $27 \mathrm{~dB}$ (in $1 \mathrm{MHz}$ RBW). The CEO frequency could be tuned by changing the current of the pump diode, but no stabilization was realized at this stage. 
A weakness of this first self-referenceable gigahertz DPSSL was the tapered pump diode. This non-commercial high-brightness source was fairly sensitive to optical feedback, which can potentially lead to a catastrophic damage of its facets [65] and resulted in a poor long-term reliability. The next breakthrough occurred when Klenner et al., demonstrated a strong CEO beat ( $30 \mathrm{~dB}$ in $100 \mathrm{kHz} \mathrm{RBW}$ ) in a gigahertz DPSSL pumped by a commercial multi-transverse-mode pump diode [20]. This achievement was the first evidence that this type of diodes could be used to pump a DPSSL for frequency comb applications, despite the higher intensity noise resulting from its highly multimode operation. This multimode noise can be transferred to the modelocked laser and ultimately to the CEO beat as shown for a TDL [31], thus making the laser more challenging to self-reference. This effect was experimentally shown by Link et al., in an Yb:KGW SESAM-modelocked DPSSL at $1040 \mathrm{~nm}$ that was alternately pumped by a spatially-multimode low-brightness $\left(M^{2} \approx 32\right)$ high-power diode array or by a single-transverse-mode high-brightness $\left(M^{2}<1.2\right)$ beam from a semiconductor vertical external cavity surface-emitting laser (VECSEL) [66]. A flipping mirror provided a simple interchange between these two sources to pump the exactly same modelocked laser with $133 \mathrm{MHz}$ repetition rate. Both pumping schemes enabled an average output power of $450 \mathrm{~mW}$ in $130 \mathrm{fs}$ pulse duration to be generated for $2 \mathrm{~W}$ of pump power. This led to a generated CEO beat with a similar SNR of more than $30 \mathrm{~dB}$ after SC generation and $f$-to- $2 f$ interferometry. However, a significantly broader linewidth of the CEO beat was observed with the low-brightness multimode pump diode, which mainly resulted from a higher noise in the frequency range of $1 \mathrm{kHz}$ to $100 \mathrm{kHz}$. According to the concept of the $\beta$-separation line introduced in Section 2.3, this higher noise occurring at high frequency implies a larger loop

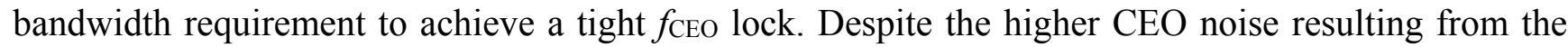
use of a low brightness multimode pump diode, Klenner et al., demonstrated that a tight CEO lock could be achieved by feedback to the current of the multi-spatial-mode pump diode [56]. An in-loop residual integrated phase noise of $407 \mathrm{mrad}[1 \mathrm{~Hz}, 5 \mathrm{MHz}$ ] was achieved in this case with a loop bandwidth of $\sim 300 \mathrm{kHz}$.

Despite their higher noise, multi-transverse-mode pump diodes offer various advantages such as the robustness and reliability of industrial-grade diodes, and power scalability achieved by increasing the number of emitters coupled to the multimode fiber. For this reason, they are also commonly used as pump sources in high-power TDLs [30,31] as will be discussed in Section 4. However, their multimode nature results in a lower brightness in comparison to tapered diodes.

In the work of Klenner et al. [20], a pump power of $13 \mathrm{~W}$ at $978 \mathrm{~nm}$ with an $M^{2}$ value of $\sim 25$ was successfully used to produce $3.4 \mathrm{~W}$ of average power at $1047 \mathrm{~nm}$ in an Yb:KGW SESAM-modelocked laser with 125 fs pulse duration and $1.06 \mathrm{GHz}$ repetition rate. The use of a $\Sigma$-shape cavity instead of the simpler Z-shape that was used in a former $\mathrm{Yb}: \mathrm{KGW}$ gigahertz laser pumped by a tapered diode [19] offered more flexibility for cavity dispersion management. A coherent octave-spanning SC spectrum was generated directly in a PCF from the output of the modelocked oscillator, without any additional pulse compression or amplification, enabling the detection of a strong CEO beat (30 $\mathrm{dB}$ $\mathrm{SNR}$ in $100 \mathrm{kHz} \mathrm{RBW}$ ) in an $f$-to- $2 f$ interferometer. However, no CEO stabilization was realized in this laser.

The first self-referenced gigahertz DPSSL was demonstrated by Klenner et al., in 2014 [21]. The gain material was changed to $\mathrm{Yb}$ :CALGO here, placed in a Z-shape cavity. Yb:CALGO has a broader emission bandwidth than $\mathrm{Yb}: \mathrm{KGW}$ and thus has the potential to produce shorter pulses. A maximum 
average output power of $1.7 \mathrm{~W}$ was generated at $1062 \mathrm{~nm}$ with $64 \mathrm{fs}$ pulse duration at a repetition rate of $1.0 \mathrm{GHz}$ by pumping the gain crystal with a commercial highly multimode fiber-coupled laser diode at $980 \mathrm{~nm}$ with an $M^{2}$ value of about 32. The maximum output power of the pump was $60 \mathrm{~W}$, but only a small fraction of $8 \mathrm{~W}$ was used in these experiments. The Z-shape cavity contained a flat output-coupler used as a folding mirror that offered two output beams with an equal output power. One of the output ports was used for SC generation and CEO detection, whereas the other one remained available for comb applications.

The high average output power available from a single output port of the laser and the short pulse duration provided a sufficient peak power for coherent octave-spanning SC generation in a $1 \mathrm{~m}$ long PCF without any amplification or pulse compression. An average power of $500 \mathrm{~mW}$ launched in the PCF followed by a quasi-common-path $f$-to- $2 f$ interferometer produced a strong CEO beat at $680 \mathrm{~nm}$ with more than $25 \mathrm{~dB}$ SNR in a RBW of $1 \mathrm{MHz}$. The free-running CEO beat had a FWHM linewidth of several hundreds of kilohertz that was significantly larger than for other formerly self-referenced DPSSLs [26,27,29], which made its stabilization more challenging. This larger linewidth was observed despite several precautions taken to minimize some potential noise sources, such as preventing optical feedback into the pump diode by slightly tilting some optical components in the pump beam, or by minimizing mechanical perturbations of the multimode fiber that can increase the relative intensity noise (RIN) of the pump diode that is ultimately transferred to CEO noise. Passive filters were also used to prevent AC noise components to enter into the standard DC power supply used to drive the pump diode, and to attenuate at the same time electrical noise from the power supply entering in the pump diode. The large free-running linewidth of the CEO beat observed in this DPSSL resulted in some part from the use of a multi-transverse-mode pump diode without wavelength-stabilizing

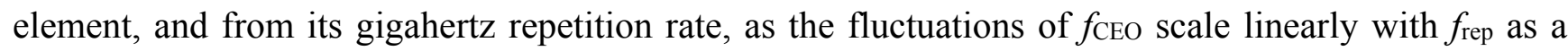
consequence of Equation (2). For this reason, high repetition rate lasers require a larger loop bandwidth to achieve a tight lock. In the Yb:CALGO laser of [21], a tight CEO lock was achieved by feedback to the pump current with a large bandwidth of $\sim 300 \mathrm{kHz}$. This was realized with a fast current modulation electronics installed in parallel to the DC power supply of the pump diode that could be directly modulated only at low frequency $(<1 \mathrm{kHz})$. The derivative part of a PID servo controller was essential to extend the overall loop bandwidth up to $\sim 300 \mathrm{kHz}$, much higher than the measured roll-off frequency of $f_{\mathrm{CEO}}$ for pump current modulation $(\sim 20-30 \mathrm{kHz})$ originating from the gain upper-state lifetime and photons lifetime in the cavity. Therefore, a tight CEO lock was successfully achieved with $\sim 300 \mathrm{kHz}$ feedback bandwidth and an in-loop residual integrated phase noise of $744 \operatorname{mrad}\left[1 \mathrm{~Hz}-5 \mathrm{MHz}\right.$. The stabilization of $f_{\text {CEO }}$ was also accompanied by a strong improvement of the RIN and of the timing jitter of the gigahertz laser in the frequency range from $\sim 100 \mathrm{~Hz}$ up to the loop bandwidth, indicating that they originate from a common noise source. This also implies that the pump current has a coupled influence on $f_{\text {CEO }}$ and $f_{\text {rep }}$, in a similar way as described in detail in the case of an Er:fiber comb [67].

A large contribution to the frequency fluctuations of $f_{\mathrm{CEO}}$ in diode-pumped femtosecond lasers usually originates from pump diode intensity noise that is transferred to $f_{\text {CEO }}$ by different mechanisms [68]. This was evidenced by McFerran et al., in an Er:fiber laser [69] and is even more pronounced in high repetition rate DPSSLs pumped by multi-transverse-mode laser diodes. In addition to the higher RIN of these pump diodes compared to a single-transverse-mode pumping scheme that 
was previously discussed, spectral fluctuations of the pump source can also induce strong $f$ CEO noise for pump lasers that do not incorporate any wavelength stabilizing element. For instance, the Yb:CALGO gain medium exhibits a quite narrow absorption peak at $980 \mathrm{~nm}$ [70]. Any wavelength fluctuation of the pump source is thus converted in fluctuations of the absorbed pump power that in turn result in $f_{\text {CEO }}$ frequency noise. Hence, a drastic improvement of the CEO stabilization was demonstrated in the aforementioned Yb:CALGO gigahertz femtosecond laser with the use of a passively wavelength-stabilized pump diode [57]. Using two different versions of the same spatially-multimode laser diode (LIMO 60-F100-DL980 from Lissotschenko Mikrooptik GmbH, Dortmund, Germany), a strong difference in the free-running CEO beat linewidth was observed. The early results previously mentioned were obtained with the simplest version of the pump diode without any wavelength-stabilizing element. The resulting pump spectrum was fluctuating in a range of about $7 \mathrm{~nm}$, much larger than the absorption peak of Yb:CALGO. On the other hand, a more evolved version of the pump diode that stabilizes the wavelength using a volume holographic grating (VHG) resulted in a stable pump spectrum with a narrow width of only $0.1 \mathrm{~nm}$ centered at $980.4 \mathrm{~nm}$, well matched to the Yb:CALGO absorption peak. In addition to its direct effect on the pump spectrum, the VHG stabilization also showed a strong improvement of the RIN of the pump source, with a reduction of $20 \mathrm{~dB}$ at all frequencies above $100 \mathrm{~Hz}$. All in all, these two effects resulted in a drastic improvement of the free-running CEO beat linewidth, with a reduction from 1.7 MHz FWHM with the unstabilized pump source down to $170 \mathrm{kHz}$ with the VHG-stabilized pump in an observation time necessary to average 100 spectra recorded on a microwave spectrum analyzer. As a result, a tight CEO lock was achieved by feedback to the pump current with a loop bandwidth slightly lower than $200 \mathrm{kHz}$. The residual integrated phase noise of only $339 \mathrm{mrad}$ is the lowest value obtained from a gigahertz DPSSL. These results clearly showed the benefit of a wavelength-stabilized pump source, but also demonstrate that a tight CEO lock can be achieved in DPSSLs with repetition rates in the gigahertz range using cost-efficient commercial multi-transverse-mode pump diodes.

Beside their potential for frequency combs with large mode spacing, Yb-DPSSLs are also attractive for very low-noise operation at lower repetition rates. A fully-stabilized frequency comb with a low residual integrated phase noise of the CEO beat of $83 \operatorname{mrad}[1 \mathrm{~Hz}-1 \mathrm{MHz}]$ has been reported by Kundermann et al. [29]. A commercial Origami-10 modelocked laser (Onefive GmbH, Zurich, Switzerland) emitting short pulses of 111 fs duration at a center wavelength of $1047 \mathrm{~nm}$ and at a repetition rate of $100 \mathrm{MHz}$ was used to generate a SC spectrum in a $50 \mathrm{~cm}$ long PCF. A CEO beat with $40 \mathrm{~dB}$ SNR in a RBW of $91 \mathrm{kHz}$ and a narrow free-running linewidth of $\sim 15 \mathrm{kHz}$ (observed in a sweep time of $461 \mathrm{~ms}$ ) was detected in an $f$-to- $2 f$ interferometer. These noise figures for a free-running comb resemble more the properties of a modelocked DPSSL than of a fiber laser, but the exact configuration of the Origami-10 femtosecond laser is unknown and was not disclosed in [29]. Nevertheless, this laser has been included in the present discussion of Yb-doped DPSSLs and its properties are also listed in Table 1. A stabilization loop with feedback applied to the current of the pump diode with a loop bandwidth of about $130 \mathrm{kHz}$ was realized, and the contribution of the locked $f_{\text {CEO }}$ to the instability of the optical carrier corresponds to a fractional frequency stability of $<10^{-17}$ at $1 \mathrm{~s}$ averaging time (with $500 \mathrm{~Hz}$ noise equivalent bandwidth).

A demanding application for frequency combs in the future is their operation in a space environment. Several missions are under consideration by space agencies that will involve a frequency 
comb, e.g., in an optical master clock in space for fundamental physics experiments, geodesy, remote comparison of high accuracy ground-based optical clocks, etc. Such frequency combs have to survive challenging environmental conditions, in particular high-energy radiation. In this context, DPSSL frequency combs constitute a valuable alternative to fiber combs that are generally the preferred choice for space applications [71]. Buchs et al. have proved the capability of a modelocked Yb:KYW DPSSL to sustain a dose of gamma and protons radiation corresponding to more than five years in outer space on a highly elliptic orbit around Earth corresponding to a possible future candidate mission for a frequency comb in space [58]. The SESAM-modelocked laser tested under radiation had an emission spectrum centered at $1029 \mathrm{~nm}$, with $148 \mathrm{~mW}$ average power emitted in 169 fs pulses at $40 \mathrm{MHz}$ repetition rate. Different parameters of the laser were recorded during the irradiation, such as the output power, repetition rate, center wavelength, etc. Some of them showed only a minor change during the gamma irradiation (e.g., only 5\% reduction of the laser output power). The initial output power was recovered a few days after the irradiation, while the recovery period was longer for the pulse width, but did not affect the overall performance of the laser.

A second similar laser with a repetition rate of $100 \mathrm{MHz}$, an average output power of $215 \mathrm{~mW}$ emitted at $1031 \mathrm{~nm}$ and a pulse duration of $155 \mathrm{fs}$ was fully-stabilized with an in-loop integrated phase noise of $f_{\text {CEO }}$ of $1.1 \mathrm{rad}(1 \mathrm{~Hz}-1 \mathrm{MHz})$ achieved with a loop bandwidth of about $100 \mathrm{kHz}$. Pulse compression to 100 fs realized using GTI mirrors after spectral broadening in a singlemode fiber was necessary for octave-spanning SC generation in a HNLF. This stabilized laser system was not subjected to irradiation.

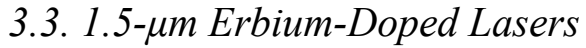

Commercial and laboratory-grade Er:fiber frequency combs are used nowadays in a variety of applications in the $1.5 \mu \mathrm{m}$ spectral range, such as low-noise microwave generation, high-resolution spectroscopy or distant comparison of optical atomic clocks via the optical fiber network. DPSSL combs are attractive for all these applications as a result of their low-noise potential, as demonstrated in the previous sub-section for Yb-based DPSSLs at $1 \mu \mathrm{m}$. However, very few DPSSL combs have been demonstrated so far in the important $1.5 \mu \mathrm{m}$ telecom range. The first self-referenceable modelocked DPSSL was reported by Stumpf et al. [72] based on an Er:Yb:glass oscillator (ERGO) pumped by a single-transverse-mode wavelength-stabilized telecom-grade diode at $976 \mathrm{~nm}$. The SESAM-modelocked laser emitted $110 \mathrm{~mW}$ of average power in $170 \mathrm{fs}$ at a repetition rate of $75 \mathrm{MHz}$. A strong CEO beat with an SNR close to $50 \mathrm{~dB}(100 \mathrm{kHz} \mathrm{RBW})$ was detected after SC generation in a polarization-maintaining HNLF and a standard $f$-to- $2 f$ interferometer. Owing to the small overall optical losses in the cavity (less than 3\%) that resulted in a high Q-factor resonator, this laser demonstrated a record narrow-linewidth CEO beat with only $4 \mathrm{kHz}$ FWHM. A tight phase lock of $f_{\mathrm{CEO}}$ was achieved by feedback to the pump current with a moderate feedback bandwidth of around $5.5 \mathrm{kHz}$ and a residual integrated phase noise of $0.72 \mathrm{rad}(0.1 \mathrm{~Hz}-100 \mathrm{kHz})$ [27]. Full stabilization of this frequency comb to an H-maser was demonstrated with a fractional frequency stability of a comb line at $1558 \mathrm{~nm}$ of $\sim 4 \times 10^{-13}$ at $1 \mathrm{~s}$, assessed from the beat signal between the comb line and a cavity-stabilized laser and limited by the stability of the RF reference used to lock $f_{\text {rep }}$ [28]. This DPSSL comb was also stabilized to an optical reference made of an ultra-stable laser for 
low-noise microwave generation and showed its benefit compared to a commercial Er:fiber comb in terms of CEO contribution to the phase noise [73].

The CEO stabilization in this ERGO laser was improved by the use of a faster actuator for $f_{\text {CEO }}$ control that enabled extending the feedback bandwidth beyond the limitation imposed by the upper-state lifetime of the gain medium. Hoffmann et al., demonstrated a new tuning and stabilization

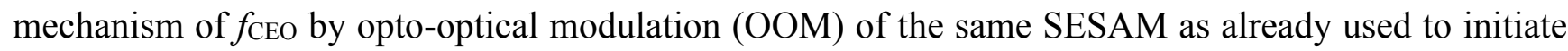
self-starting modelocking in this laser using a continuous wave pump laser at $812 \mathrm{~nm}$ [44]. The method combines high feedback bandwidth with low loss, low nonlinearity, and low dispersion, which makes it power-scalable and very attractive for high power oscillators such as TDLs. A drastic improvement of the stabilized CEO beat in the ERGO comb was achieved compared to pump current modulation, in particular a fractional frequency instability of $f_{\text {CEO reduced by a factor of four and a residual integrated }}$ phase noise of only $63 \mathrm{mrad}(1 \mathrm{~Hz}-100 \mathrm{kHz})$, or even less than $30 \mathrm{mrad}$ when technical noise at $50 \mathrm{~Hz}$ and harmonics was removed. This integrated phase noise is the lowest value reported so far for any DPSSL comb to the best of our knowledge and clearly proves the capability of DPSSL combs for low-noise operation.

A comparison between different approaches for $f_{\text {CEO }}$ stabilization in a frequency comb generated from a similar Er:Yb:glass DPSSL was reported by Kundermann and Lecomte [59]. The output spectrum of a commercial Origami-15 Er:Yb:glass DPSSL (Onefive GmbH, Zurich, Switzerland) with $190 \mathrm{fs}$ transform-limited pulses at $1560 \mathrm{~nm}$ and $100 \mathrm{MHz}$ repetition rate with an average output power of $115 \mathrm{~mW}$ was spectrally broadened in a HNLF. A CEO beat was then detected in an $f$-to- $2 f$ interferometer. The laser was pumped by a singlemode fiber-coupled Bragg-grating-stabilized diode at $976 \mathrm{~nm}$ and previously demonstrated state-of-the-art amplitude noise and sub-100 attoseconds integrated timing jitter $(10 \mathrm{kHz}-50 \mathrm{MHz})$ [74]. CEO stabilization using feedback to an extra-cavity acousto-optic frequency shifter (AOFS) was shown to achieve an integrated phase noise of $118 \mathrm{mrad}$

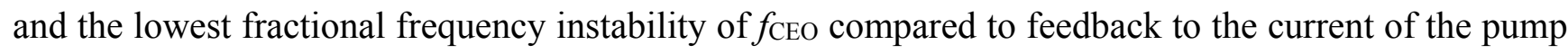
diode and to a feed-forward control of $f_{\text {CEO }}$ using the AOFS. Two similar DPSSLs were successfully applied for ultra-low-noise microwave generation using five-stage pulse interleavers to multiply the repetition rate of the detected pulse trains to $3.2 \mathrm{GHz}$ [75] and for dual-comb spectroscopy in a range between 1.55 and $2.25 \mu \mathrm{m}$ after spectral broadening in an HNLF [76].

\section{Self-Referenced DPSSLs in Thin Disk Geometry}

TDLs constitute a particular DPSSL technology where the gain medium is made of a very thin disk-shaped crystal (typical thickness of a few hundred micrometers) instead of the mm-size bulk gain medium commonly used in conventional DPSSLs previously discussed in Section 3. The disk thickness is much smaller than the laser beam diameter, so that the heat generated by pump absorption is principally extracted in the longitudinal direction through the backside of the disk rather than transversely. This geometry first introduced in the early 1990s provides an efficient cooling of the disk from the back side that leads to straightforward power scalability [77]. As for DPSSLs with a bulk gain medium, SESAM-modelocking and KLM are the main modelocking mechanisms used in TDLs. SESAM-modelocked TDLs enable direct power scaling by increasing the beam size on the thin disk gain medium and on the SESAM, without detrimental increase of the nonlinearities. As a result, TDLs 
are able to reach high power operation and Yb:YAG SESAM-modelocked lasers have demonstrated the highest average power ( $275 \mathrm{~W}$ in $583 \mathrm{fs}$ pulses) and pulse energy ( $80 \mu \mathrm{J}$ in $1.1 \mathrm{ps}$ pulses) of any type of modelocked oscillators [36,78]. Therefore, TDLs are well-suited as driving sources for experiments requiring high intensities at megahertz repetition rates, e.g., to generate vacuum ultra-violet (VUV) or extreme ultra-violet (XUV) radiation by high harmonic generation (HHG). Today, TDLs are developed mainly in the $1 \mu \mathrm{m}$ spectral range based on Yb-doped disks, such as Yb:YAG.

The small disk thickness results in an inefficient pump absorption in the gain medium. Therefore, a multi-pass pump arrangement is generally used, where a parabolic mirror combined with prism retroreflectors enables for typically 8,16 or 24 double passes of the pump radiation through the disk without stringent requirements on the pump beam quality. For this purpose, the back side of the disk has a highly reflective coating for both pump and laser wavelengths, whereas the front side is anti-reflection coated. TDLs typically use high-power laser diode bars coupled into a highly multi-transverse-mode optical fiber (with a typical $M^{2}$ value $>100$ ) and require current drivers operating at several tens of amperes. For a long time, it was not clear if this pumping scheme introduces too high noise for comb self-referencing.

The first CEO beat detection in a TDL was achieved by Saraceno et al., in 2011 [60]. The SESAM-modelocked $\mathrm{Yb}: \mathrm{Lu}_{2} \mathrm{O}_{3}$ TDL emitted $7 \mathrm{~W}$ of average output power in pulses of $142 \mathrm{fs}$ duration at $64 \mathrm{MHz}$ repetition rate. The laser was pumped with $47 \mathrm{~W}$ at $976 \mathrm{~nm}$ delivered by a VHG-stabilized diode with 24 passes on the disk. The very short pulses emitted by this TDL enabled SC generation in a PCF using less than $1 \%$ of the laser output power without any pulse amplification or compression. The low soliton order $N_{\text {sol }}=5$ of the pulses launched into the PCF led to a coherent SC according to the argumentation presented in Section 2.2. A CEO beat with an SNR $>30 \mathrm{~dB}$ in a RBW of $1 \mathrm{kHz}$ was detected in an $f$-to- $2 f$ interferometer. Tuning of $f_{\text {CEO }}$ with the current of the pump diode was observed in this TDL, but no self-referencing was performed due to the damage of the only crystal available at the time of the experiment.

The first self-referenced TDL was demonstrated by Pronin et al. [61]. The KLM Yb:YAG laser delivered an average output power of $17 \mathrm{~W}$ at a center wavelength of $1030 \mathrm{~nm}$ in $250 \mathrm{fs}$ pulses and $38 \mathrm{MHz}$ repetition rate. It was pumped by $110 \mathrm{~W}$ from a fiber-coupled diode at $940 \mathrm{~nm}$ with 24 passes on the disk. The output pulses were compressed to $30 \mathrm{fs}$ using chirped mirrors before being launched into an $f$-to- $2 f$ interferometer that included a $8 \mathrm{~cm}$ long PCF for SC generation. Two identical interferometers were built to provide in-loop and out-of-loop measurements. The free-running CEO beat was detected with an SNR of about $35 \mathrm{~dB}$ in a RBW of $300 \mathrm{kHz}$. The CEO frequency had a high sensitivity to the pump current owing to the strong amplitude-to-phase coupling induced in the silica plate Kerr medium. Therefore, pump current modulation was first implemented for CEO-stabilization. With the use of a phase-lead filter to overcome the measured $3 \mathrm{~dB}$ roll-off frequency of $3 \mathrm{kHz}$ in the response of $f_{\text {CEO }}$ to a modulation of the pump current, a residual integrated in-loop phase noise of $250 \mathrm{mrad}$ was achieved $(1 \mathrm{~Hz}-500 \mathrm{kHz})$. Improvement of the CEO stabilization was obtained using direct laser intra-cavity power modulation via an acousto-optic modulator (AOM) placed inside the resonator to overcome the limitation of the $1 \mathrm{~ms}$ long lifetime of $\mathrm{Yb}$ and the correspondingly low achievable servo-loop bandwidth. In this case a residual integrated phase noise of 100 mrad in-loop 
and $200 \mathrm{mrad}$ out-of-loop were reported. However, the use of an intra-cavity AOM appears more difficult to be implemented in TDLs with higher intra-cavity power due to thermal issues.

A refinement of these initial results including a more thorough characterization of the CEO stabilization was recently reported by the same group [62]. The output power of the oscillator was increased to $40 \mathrm{~W}$ and the external compression reduced the pulse duration to $17 \mathrm{fs}$ after a first stage of spectral broadening/compression and down to $7.7 \mathrm{fs}$ using tailored dispersion mirrors in a second stage. Due to the bandwidth limitation of the CEO control by the pump current ( $3 \mathrm{~dB}$ point of $1.5 \mathrm{kHz})$ resulting from the $1 \mathrm{~ms}$ gain relaxation time, stabilization with the intra-cavity AOM was preferred. The AOM placed at the Brewster angle within the cavity was used as a loss modulator with a maximum modulation amplitude of $2 \%$. In-loop integrated phase noise of $180 \mathrm{mrad}$ was achieved, and $270 \mathrm{mrad}$ out-of-loop $(1 \mathrm{~Hz}-500 \mathrm{kHz})$. This difference was partly attributed to amplitude-to-phase (AM-to-PM) noise conversion in the SC generation process and partly to the digital phase detector used in the CEO loop, possibly resulting from nonlinearities in the behavior of this device according to the observations reported in [45].

The first SESAM-modelocked TDL was reported by Klenner et al. [30]. The Yb:CALGO TDL generated $90 \mathrm{fs}$ pulses at $65 \mathrm{MHz}$ repetition rate with an average output power of $2.1 \mathrm{~W}$ at a center wavelength of $1052 \mathrm{~nm}$. A pump laser diode made of two diode stacks VHG-stabilized to a wavelength of $979.5 \mathrm{~nm}$ with a maximum output power of $400 \mathrm{~W}$ was used to pump the gain medium in an arrangement with 24 passes over the disk. A fraction of only $65 \mathrm{~W}$ of pump power was used in the reported results, which was coupled into a multimode fiber with $400 \mu \mathrm{m}$ diameter and an $M^{2}$ value of about 140 . The sub-100 fs pulse duration of this laser was among the shortest produced directly from a TDL oscillator at this time and was made possible by the broadband gain of the Yb:CALGO crystal. Consequently, no external pulse compression was needed for SC generation and subsequent CEO beat detection in a quasi-common-path $f$-to- $2 f$ interferometer. A fraction of about only $2 \%$ of the laser output power was used for SC generation in a PCF, resulting into a soliton order $N_{\text {sol }}=5$ that supported the generation of a coherent octave-spanning SC spectrum resulting in the detection of a CEO beat signal at $680 \mathrm{~nm}$ with $33 \mathrm{~dB}$ SNR in a RBW of $100 \mathrm{kHz}$. A tight CEO lock was achieved by feedback to the pump current with a loop bandwidth of $30-40 \mathrm{kHz}$. This was implemented using in-house designed modulation electronics capable of applying a small modulation with a bandwidth of about $100 \mathrm{kHz}$ on top of the laser DC current delivered by a standard power supply. An in-loop integrated phase noise of $120 \mathrm{mrad}$ was achieved for the tightly locked CEO beat, resulting in an rms frequency fluctuation of $f_{\mathrm{CEO}}$ of $8.8 \mathrm{mHz}$ observed over a period of $17 \mathrm{~min}$ for the $26 \mathrm{MHz}$ CEO frequency, corresponding to a contribution $<10^{-16}$ to the frequency instability of an optical comb line.

The first CEO beat detection in a TDL operating in a high average power regime of $>100 \mathrm{~W}$ was reported by Diebold et al. [63]. The SESAM-modelocked Yb:YAG TDL with a similar design to the record-high average power TDL [36] was operated in a low-pressure (150 mbar) helium environment. It delivered $140 \mathrm{~W}$ of average power at $9.67 \mathrm{MHz}$ with $\sim 750 \mathrm{fs}$ pulses. With this long pulse duration, external compression was necessary to achieve sufficiently short pulses for coherent SC generation. A small fraction of the oscillator output power was thus compressed down to $63 \mathrm{fs}$ by combining spectral broadening by self-phase modulation in a large-mode-area PCF and Gires-Tournois-interferometer (GTI) mirrors. A strong CEO beat with $>33 \mathrm{~dB}$ SNR in a RBW of $100 \mathrm{kHz}$ was then detected in a commercially-available $f$-to- $2 f$ interferometer. Whereas a significant shift of $f_{\text {CEO }}$ with the pump power 
was observed in this laser, which should enable self-referencing by the common method of pump power modulation, the CEO stabilization could not be achieved.

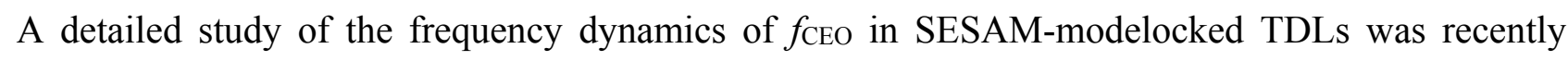
reported by Emaury et al., where the behavior of the aforementioned Yb:CALGO laser with $65 \mathrm{MHz}$ repetition rate and high-power $\mathrm{Yb}$ :YAG laser with sub-10 $\mathrm{MHz}$ repetition rate was compared [31]. A complete noise characterization of these two laser systems including the measurements of various relevant transfer functions combined with comparison with theoretical models evidenced a fundamentally different behavior of the two TDL resonators. This difference led to the impossibility to CEO-stabilize the high power Yb:YAG oscillator. Even if a comparable RIN of the multi-transverse-mode pump diodes used in these two systems was identified as the main contribution to the CEO noise in each case, the laser cavity dynamics was shown to play an essential role in the CEO frequency dynamics. Whereas the transfer function of $f_{\text {CEO }}$ for pump modulation was characterized by a highly damped second-order low-pass filter with a cut-off frequency of $\sim 12 \mathrm{kHz}$ and a smooth associated phase change in the $\mathrm{Yb}$ :CALGO TDL, it behaved like a resonant second-order filter in the Yb:YAG laser with a pronounced resonance at $7.5 \mathrm{kHz}$. The abrupt phase change associated to this resonance that occurred in a narrow frequency range led to the impossibility to achieve a tight phase lock of $f_{\text {CEO in }}$ this laser. The different operation point of the SESAMs used for modelocking of these TDLs was identified as the main cause for the different damping factor observed in the cavity transfer functions. More precisely, the relevant parameter was the variation of the SESAM nonlinear loss as a function of the intra-cavity pulse energy. Experimental results were in good agreement with theoretical considerations derived from the complex cavity transfer function obtained for modelocked lasers [79]. As an outcome of this analysis, a laser cavity exhibiting a high damping of the relaxation oscillation by nonlinear intra-cavity elements, for example by operating the SESAM in the roll-over regime, is required for CEO stabilization. This work presented important guidelines towards the future frequency stabilization of multi-100-W TDL oscillators. The importance of the CEO dynamics in the feasibility to achieve a tight CEO lock in a frequency comb shown in the case of these TDLs complements previous observations reported in Section 2.3 for a bulk Er:Yb:glass DPSSL. Alternative stabilization methods that do not use pump current modulation may be used instead to circumvent the TDL cavity transfer function, such as using SESAM OOM for CEO stabilization as previously reported in a DPSSL [44]. The extremely low losses of SESAMs make this technology compatible with high-power and high-energy lasers such as TDLs. Therefore, SESAM OOM appears as a well-suited complementary approach for CEO stabilization of TDLs.

\section{Conclusions and Outlook}

In the first years after their invention, CEO-stable laser systems were either based on Ti:Sapphire or fiber lasers. However, in the last years, CEO-stabilized DPSSLs have become a promising alternative for frequency comb generation with state-of-the-art performance. The DPSSL technology combines the advantages of fiber lasers in terms of simplicity and reliable diode pumping with the low-noise operation of Ti:Sapphire lasers resulting from their high Q-factor resonator. SESAM-modelocked DPSSLs provide self-starting and reliable operation. Their simple pumping using laser diodes provides a reliable scheme for frequency comb CEO-stabilization using the common method of feedback to the 
current of the pump diode. The lower noise of DPSSLs arising from their low cavity losses results in less demanding requirements in terms of feedback bandwidth for CEO stabilization compared to fiber lasers. A tight CEO lock has been demonstrated in various DPSSL systems using this simple feedback scheme, despite the limited bandwidth achievable with this approach resulting from the relatively long upper-state lifetime of the gain material. Faster actuators for improved CEO stabilization have also been demonstrated to control and stabilize $f_{\text {CEO }}$ with a higher bandwidth, such as by opto-optical modulation of an intra-cavity SESAM [44] or using an intra-cavity AOM in TDLs [61].

The superior emission properties of DPSSLs in terms of average output power and pulses duration also enable CEO detection directly from the output of the laser oscillator in various systems, without the need for any external amplification or pulse compression, which keeps the system more simple and cost-efficient. DPSSL frequency combs will certainly continue their expansion in the near future, especially for the realization of frequency combs with multi-gigahertz repetition rates, a range that is difficult to achieve for fiber lasers. High repetition rate combs are of particular interest for applications such as ultra-low-noise microwave generation or astronomical spectrographs calibration. First experiments of ultra-low-noise microwave generation using DPSSL combs with repetition rates in the $100 \mathrm{MHz}$ range were reported with very promising performance [26,73,75], and it is likely that further applications will develop in the future that will benefit from the low-noise properties and high repetition rate capabilities of DPSSLs. Such gigahertz DPSSL combs can be pumped using commercial multi-transverse-mode diodes that make these laser systems cost-efficient and reliable, without compromising the achievement of a tight CEO lock [21]. DPSSLs have also shown their hardness to radiation [58], which makes them an attractive alternative to fiber lasers for the implementation of frequency combs in a space environment, for instance for a future master optical clock in space.

Another field where DPSSLs are already in a leading position is high-power ultrafast oscillators. The TDL geometry has currently demonstrated the highest average power and pulse energy emitted from any oscillator [36,78]. New breakthroughs in this domain are expected in the near future for the generation of stabilized frequency combs in the high average power regime of $>100 \mathrm{~W}$, which will be of paramount importance for high field physics such as high harmonic generation to produce extreme ultra-violet frequency combs.

\section{Acknowledgments}

We acknowledge support by the Swiss National Science Foundation (SNF) project 146738, the European Research Council (ERC Starting Grant 2011 \#279545), and Nano-Tera.ch MIXSEL-2 (20NA21_145932).

\section{Conflicts of Interest}

The authors declare no conflict of interest.

\section{References}

1. Hänsch, T.W. Nobel lecture: Passion for precision. Rev. Mod. Phys. 2006, 78, 1297-1309. 
2. Ye, J.; Schnatz, H.; Hollberg, L.W. Optical frequency combs: From frequency metrology to optical phase control. IEEE J. Sel. Top. Quantum Electron. 2003, 9, 1041-1058.

3. Diddams, S.A.; Udem, T.; Bergquist, J.C.; Curtis, E.A.; Drullinger, R.E.; Hollberg, L.; Itano, W.M.; Lee, W.D.; Oates, C.W.; Vogel, K.R.; et al. An Optical Clock Based on a Single Trapped ${ }^{199} \mathrm{Hg}^{+}$Ion. Science 2001, 293, 825-828.

4. Sterr, U.; Degenhardt, C.; Stoehr, H.; Lisdat, C.; Schnatz, H.; Helmcke, J.; Riehle, F.; Wilpers, G.; Oates, C.; Hollberg, L. The optical calcium frequency standards of PTB and NIST. C. R. Phys. 2004, 5, 845-855.

5. Quinlan, F.; Fortier, T.M.; Kirchner, M.S.; Taylor, J.A.; Thorpe, M.J.; Lemke, N.; Ludlow, A.D.; Jiang, Y.; Diddams, S.A. Ultralow phase noise microwave generation with an Er:fiber-based optical frequency divider. Opt. Lett. 2011, 36, 3260-3262.

6. Diddams, S.A.; Hollberg, L.; Mbele, V. Molecular fingerprinting with the resolved modes of a femtosecond laser frequency comb. Nature 2007, 445, 627-630.

7. Schiller, S. Spectrometry with frequency combs. Opt. Lett. 2002, 27, 766-768.

8. Diddams, S.A. The evolving optical frequency comb. J. Opt. Soc. Am. B 2010, 27, B51-B62.

9. Ma, L.S.; Zucco, M.; Picard, S.; Robertsson, L.; Windeler, R.S. A new method to determine the absolute mode number of a mode-locked femtosecond-laser comb used for absolute optical frequency measurements. IEEE J. Sel. Top. Quantum Electron. 2003, 9, 1066-1071.

10. Hall, J.L. Nobel lecture: Defining and measuring optical frequencies. Rev. Mod. Phys. 2006, 778, 1279-1295.

11. Telle, H.R.; Steinmeyer, G.; Dunlop, A.E.; Stenger, J.; Sutter, D.H.; Keller, U. Carrier-envelope offset phase control: A novel concept for absolute optical frequency measurement and ultrashort pulse generation. Appl. Phys. B 1999, 69, 327-332.

12. Holzwarth, R.; Udem, T.; Hänsch, T.W.; Knight, J.C.; Wadsworth, W.J.; Russell, P.S. Optical frequency synthesizer for precision spectroscopy. Phys. Rev. Lett. 2000, 85, 2264-2267.

13. Jones, D.J.; Diddams, S.A.; Ranka, J.K.; Stentz, A.; Windeler, R.S.; Hall, J.L.; Cundiff, S.T. Carrier envelope phase control of femtosecond mode-locked lasers and direct optical frequency synthesis. Science 2000, 288, 635-639.

14. Predehl, K.; Grosche, G.; Raupach, S.M.F.; Droste, S.; Terra, O.; Alnis, J.; Legero, T.; Hänsch, T.W.; Udem, T.; Holzwarth, R.; et al. A 920-Kilometer Optical Fiber Link for Frequency Metrology at the 19th Decimal Place. Science 2012, 336, 441-444.

15. Hartl, I.; McKay, H.A.; Thapa, R.; Thomas, B.K.; Ruehl, A.; Dong, L.; Fermann, M.E. Fully Stabilized GHz Yb-Fiber Laser Frequency Comb. In Proceedings of the Advanced Solid-State Photonics; Denver, CO, USA, 1-4 February 2009.

16. Lee, C.-C.; Mohr, C.; Bethge, J.; Suzuki, S.; Fermann, M.E.; Hartl, I.; Schibli, T.R. Frequency comb stabilization with bandwidth beyond the limit of gain lifetime by an intracavity graphene electro-optic modulator. Opt. Lett. 2012, 37, 3084-3086.

17. Klenner, A.; Golling, M.; Keller, U. High peak power gigahertz Yb:CALGO laser. Opt. Express 2014, 22, 11884-11891.

18. Pekarek, S.; Klenner, A.; Südmeyer, T.; Fiebig, C.; Paschke, K.; Erbert, G.; Keller, U. Femtosecond diode-pumped solid-state laser with a repetition rate of $4.8 \mathrm{GHz}$. Opt. Express 2012, $20,4248-4253$. 
19. Pekarek, S.; Südmeyer, T.; Lecomte, S.; Kundermann, S.; Dudley, J.M.; Keller, U. Self-referenceable frequency comb from a gigahertz diode-pumped solid-state laser. Opt. Express 2011, 19, 16491-16497.

20. Klenner, A.; Golling, M.; Keller, U. A gigahertz multimode-diode-pumped Yb:KGW enables a strong frequency comb offset beat signal. Opt. Express 2013, 21, 10351-10357.

21. Klenner, A.; Schilt, S.; Südmeyer, T.; Keller, U. Gigahertz frequency comb from a diode-pumped solid-state laser. Opt. Express 2014, 22, 31008-31019.

22. Fortier, T.M.; Kirchner, M.S.; Quinlan, F.; Taylor, J.; Bergquist, J.C.; Rosenband, T.; Lemke, N.; Ludlow, A.; Jiang, Y.; Oates, C.W.; et al. Generation of Ultrastable Microwaves via Optical Frequency Division. Nat. Photonics 2011, 5, 425-429.

23. Steinmetz, T.; Wilken, T.; Araujo-Hauck, C.; Holzwarth, R.; Hänsch, T.W.; Pasquini, L.; Manescau, A.; D’Odorico, S.; Murphy, M.T.; Kentischer, T.; et al. Laser Frequency Combs for Astronomical Observations. Science 2008, 321, 1335-1337.

24. Rauschenberger, J.; Fortier, T.; Jones, D.; Ye, J.; Cundiff, S. Control of the frequency comb from a modelocked Erbium-doped fiber laser. Opt. Express 2002, 10, 1404-1410.

25. Meyer, S.A.; Squier, J.A.; Diddams, S.A. Diode-pumped Yb:KYW femtosecond laser frequency comb with stabilized carrier envelope offset frequency. Eur. Phys. J. D 2008, 48, 19-26.

26. Meyer, S.A.; Fortier, T.M.; Lecomte, S.; Diddams, S.A. A frequency-stabilized Yb:KYW femtosecond laser frequency comb and its application to low-phase-noise microwave generation. Appl. Phys. B 2013, 112, 565-570.

27. Schilt, S.; Bucalovic, N.; Dolgovskiy V.; Schori, C.; Stumpf, M.C.; di Domenico, G.; Pekarek, S.; Oehler, A.E.H.; Südmeyer, T.; Keller, U.; et al. Fully stabilized optical frequency comb with sub-radian CEO phase noise from a SESAM modelocked 1.5- $\mu \mathrm{m}$ solid-state laser. Opt. Express 2011, 19, 24171-24181.

28. Schilt, S.; Dolgovski, V.; Bucalovic, N.; Schori, C.; Stumpf, M.C.; Di Domenico, G.; Pekarek, S.; Oehler, A.E.H.; Südmeyer, T.; Keller, U.; et al. Noise properties of an optical frequency comb from a SESAM-modelocked $1.5-\mu \mathrm{m}$ solid-state laser stabilized to the $10^{-13}$ level, Appl. Phys. B 2012, 109, 391-402.

29. Kundermann, S.; Portuondo-Campa, E.; Lecomte, S. Ultra-low-noise $1 \mu \mathrm{m}$ optical frequency comb. IEEE Electron. Lett. 2014, 50, 1231-1232

30. Klenner, A.; Emaury, F.; Schriber, C.; Diebold, A.; Saraceno, C.J.; Schilt, S.; Keller, U.; Südmeyer, T. Phase-Stabilization of the Carrier-Envelope-Offset Frequency of a SESAM modelocked Thin Disk Laser. Opt. Express 2013, 21, 24770-24780.

31. Emaury, F.; Diebold, A.; Klenner, A.; Saraceno, C.J.; Schilt, S.; Südmeyer, T.; Keller, U. Frequency comb offset dynamics of SESAM modelocked thin disk lasers. Opt. Express 2015, 23, 21836-21856.

32. Kippenberg, T.J.; Holzwarth, R.; Diddams, S.A. Microresonator-based optical frequency combs. Science 2011, 332, 555-559.

33. Del'Haye, P.; Arcizet, O.; Schliesser, A.; Holzwarth, R.; Kippenberg, T.J. Full Stabilization of a Microresonator-Based Optical Frequency Comb. Phys. Rev. Lett. 2008, 101, 053903.

34. Hou, D.; Ning, B.; Wu, J.; Wang, Z.; Zhao, J. Demonstration of a stable erbium-fiber-laser-based frequency comb based on a single rubidium atomic resonator. Appl. Phys. Lett. 2013, 102, 151104. 
35. Schratwieser, T.C.; Balskus, K.; McCracken, R.A.; Farrell, C.; Leburn, C.G.; Zhang, Z.; Lamour, T.P.; Ferreiro, T.I.; Marandi, A.; Arnold, A.S.; et al. ${ }^{87} \mathrm{Rb}$-stabilized 375-MHz Yb:fiber femtosecond frequency comb. Opt. Express 2014, 22, 10494-10499.

36. Saraceno, C.J.; Emaury, F.; Heckl, O.H.; Baer, C.R.E.; Hoffmann, M.; Schriber, C.; Golling, M.; Südmeyer, T.; Keller, U. $275 \mathrm{~W}$ average output power from a femtosecond thin disk oscillator operated in a vacuum environment. Opt. Express 2012, 20, 23535-23541.

37. Ferray, M.; L’Huillier, A.; Li, X.F.; Lompré, L.A.; Mainfray, G.; Manus, C. Multiple-harmonic conversion of $1064 \mathrm{~nm}$ radiation in rare gases. J. Phys. B 1988, 21, L31-L35.

38. Apolonski, A.; Poppe, A.; Tempea, G.; Spielmann, C.; Udem, T.; Holzwarth, R.; Hänsch, T.W.; Krausz, F. Controlling the Phase Evolution of Few-Cycle Light Pulses. Phys. Rev. Lett. 2000, 85, 740-743.

39. Ramond, T.M.; Diddams, S.A.; Hollberg, L.; Bartels, A. Phase coherent link from optical to microwave frequencies via the broadband continuum from a $1 \mathrm{GHz}$ Ti:Sapphire femtosecond oscillator. Opt. Lett. 2002, 27, 1842-1844.

40. Ell, R.; Morgner, U.; Kärtner, F.X.; Fujimoto, J.G.; Ippen, E.P.; Scheuer, V.; Angelow, G.; Tschudi, T.; Lederer, M.J.; Boiko A.; et al. Generation of 5 fs pulses and octave-spanning spectra directly from a Ti:Sapphire laser. Opt. Lett. 2001, 26, 373-375.

41. Dudley, J.M.; Genty, G.; Cohen, S. Supercontinuum generation in photonic crystal fiber. Rev. Mod. Phys. 2006, 78, 1135-1184.

42. Meyer, A.S.; Klenner, A.; Johnson, A.R.; Luke, K.; Lamont, M.R.E.; Okawachi, Y.; Lipson, M.; Gaeta, A.L.; Keller, U. Frequency comb offset detection using supercontinuum generation in silicon nitride waveguides. Opt. Express 2015, 23, 15440-15451.

43. Kim, K.; Washburn, B.R.; Wilpers, G.; Oates, C.W.; Hollberg, L.; Newbury, N.R.; Diddams, S.A.; Nicholson, J.W.; Yan, M.F. Stabilized frequency comb with a self-referenced femtosecond Cr:forsterite laser. Opt. Lett. 2005, 30, 932-934.

44. Hoffmann, M.; Schilt, S.; Südmeyer, T. CEO stabilization of a femtosecond laser using a SESAM as fast opto-optical modulator. Opt. Express 2013, 21, 30054-30064.

45. Schilt, S.; Bucalovic, N.; Tombez, L.; Dolgovskiy, V.; Schori, C.; Di Domenico, G.; Zaffalon, M.; Thomann, P. Frequency discriminators for the characterization of narrow-spectrum heterodyne beat signals: Application to the measurement of a sub-hertz carrier-envelope-offset beat in an optical frequency comb. Rev. Sci. Instrum. 2011, 82, 123116.

46. Riehle, F. Frequency Standards, Basics and Applications; WILEY-VCH Verlag GmbH \& Co.: Weinheim, Germany, 2004; Chapter 3.

47. Di Domenico, G.; Schilt, S.; Thomann, P. Simple approach to the relation between laser frequency noise and laser lineshape. Appl. Opt. 2010, 49, 4801-4807.

48. Bucalovic, N.; Dolgovskiy, V.; Schori, C.; Thomann, P.; Di Domenico, G.; Schilt, S. Experimental Validation of a Simple Approximation to Determine the Linewidth of a Laser from Its Frequency Noise Spectrum. Appl. Opt. 2012, 51, 4582-4588.

49. Bucalovic, N.; Dolgovskiy, V.; Stumpf, M.C.; Schori, C.; di Domenico, G.; Keller, U.; Schilt, S.; Südmeyer, T. Effect of the Carrier-Envelope-Offset Dynamics on the Stabilization of a Diode-Pumped Solid-State Frequency Comb. Opt. Lett. 2012, 37, 4428-4430. 
50. Fox, R.W.; Oates, C.W.; Hollberg, L.W. Stabilizing diode lasers to high-finesse cavities; In Experimental Methods in the Physical Sciences; Cavity-Enhanced Spectroscopies; van Zee, R.D., Looney, J.P., Eds.; Academic Press: Waltham, MA, USA, 2003; Volume 40, pp. $1-46$.

51. Cundiff, S.T. Phase stabilization of ultrashort optical pulses. J. Phys. D Appl. Phys. 2002, 35, R43-R57.

52. Koke, S.; Grebing, C.; Frei, H.; Anderson, A.; Assion, A.; Steinmeyer, G. Direct frequency comb synthesis with arbitrary offset and shot-noise-limited phase noise. Nat. Photonics 2010, 4, $462-465$.

53. Krauss, G.; Fehrenbacher, D.; Brida, D.; Riek, C.; Sell, A.; Huber, R.; Leitenstorfer, A. All-passive phase locking of a compact Er:fiber laser system. Opt. Lett. 2011, 36, 540-542.

54. Keller, U.; Weingarten, K.J.; Kärtner, F.X.; Kopf, D.; Braun, B.; Jung, I.D.; Fluck, R.; Hönninger, C.; Matuschek, N.; Aus der Au, J. Semiconductor saturable absorber mirrors (SESAMs) for femtosecond tonanosecond pulse generation in solid-state lasers. IEEE J. Sel. Top. Quantum Electron. 1996, 2, 435-453.

55. Holzwarth, R.; Zimmermann, M.; Udem, T.; Hänsch, T.W.; Russbüldt, P.; Gäbel, K.; Poprawe, R.; Knight, J.C.; Wadsworth, W.J.; Russell, P.S.J. White-light frequency comb generation with a diode-pumped Cr:LiSAF laser. Opt. Lett. 2001, 26, 1376-1378.

56. Klenner, A.; Emaury, F.; Diebold, A.; Schriber, C.; Saraceno, C.J.; Schilt, S.; Südmeyer, T.; Keller, U. Harnessing frequency combs pumped by multimode high-power diodes. In Proceedings of the EPS-QEOD Europhoton Conference, Neuchatel, Switzerland, 24-29 August 2014.

57. Meyer, A.S.; Klenner, A.; Johnson, A.R.; Luke, K.; Lamont, M.R.E.; Okawachi, Y.; Lipson, M.; Gaeta, A.L.; Keller, U. Low-noise Gigahertz Frequency Comb from diode-Pumped Solid-State Laser using Silicon Nitride Waveguides. In Proceedings of the Advanced Solid State Lasers Conference and Exhibition (ASSL), Berlin, Germany, 4-8 October 2015.

58. Buchs, G.; Kundermann, S.; Portuondo-Campa, E.; Lecomte, S. Radiation hard mode-locked laser suitable as a spaceborne frequency comb. Opt. Express 2015, 23, 9890-9900.

59. Kundermann, S.; Lecomte, S. Comparison of different carrier-envelope frequency stabilization methods for a high performance DPSSL frequency comb. In Proceedings of the 2015 Joint Conference of the IEEE International Frequency Control Symposium \& European Frequency and Time Forum (FCS), Denver, CO, USA, 12-16 April 2015; pp. 594-598.

60. Saraceno, C.J.; Pekarek, S.; Heckl, O.H.; Baer, C.R.E.; Schriber, C.; Golling, M.; Beil, K.; Kränkel, C.; Huber, G.; Keller, U.; et al. Self-referenceable frequency comb from an ultrafast thin disk laser. Opt. Express 2012, 20, 9650-9656.

61. Pronin, O.; Seidel, M.; Brons, J.; Lücking, F.; Pervak, V.; Apolonski, A.; Udem, T.; Krausz, F. Carrier-envelope phase stabilized thin-disk oscillator. In Proceedings of the Advanced Solid-State Lasers conference, Paris, France, 27 October-1 November 2013.

62. Pronin, O.; Seidel, M.; Lücking, F.; Brons, J.; Fedulova, E.; Trubetskov, M; Pervak, V.; Apolonski, A.; Udem, T.; Krausz, F. High-power multi-megahertz source of waveform-stabilized few-cycle light. Nat. Commun. 2015, 6, 6988. 
63. Diebold, A.; Emaury, F.; Schriber, C.; Saraceno, C.J.; Südmeyer, T.; Keller, U. Self-referenceable frequency comb with $140 \mathrm{~W}$ of average power. In Proceedings of the 6th EPS-QEOD Europhoton Conference, Neuchatel, Switzerland, 24-29 August 2014.

64. Hönninger, C.; Paschotta, R.; Morier-Genoud, F.; Moser, M.; Keller, U. Q-switching stability limits of continuous-wave passive mode locking. J. Opt. Soc. Am. B 1999, 16, 46-56.

65. Chin, A.K.; Bertaska, R.K. Catastrophic Optical Damage in High-Power, Broad-Area Laser Diodes. In Materials and Reliability Handbook for Semiconductor Optical and Electron Devices; Ueda, O., Pearton, S.J., Eds.; Springer: New York, NY, USA, 2013; pp. 123-147.

66. Link, S.M.; Klenner, A.; Mangold, M.; Zaugg, C.A.; Mayer, A.S.; Gini, E.; Tilma, B.W.; Keller, U. Passive linewidth reduction of the carrier-envelope-offset frequency. In Proceedings of the 6th EPS-QEOD Europhoton Conference, Neuchatel, Switzerland, 24-29 August 2014.

67. Dolgovskiy, V.; Bucalovic, N.; Thomann, P.; Schori, C.; Di Domenico, G.; Schilt, S. Cross-influence between the two servo-loops of a fully-stabilized Er:fiber optical frequency comb. J. Opt. Soc. Am. B 2012, 29, 2944-2957.

68. Mulder, T.D.; Scott, R.P.; Kolner, B.H. Amplitude and envelope phase noise of a modelocked laser predicted from its noise transfer function and the pump noise power spectrum. Opt. Express 2008, 16, 14186-14191.

69. McFerran, J.J.; Swann, W.C.; Washburn, B.R.; Newbury, N.R. Suppression of pump-induced frequency noise in fiber-laser frequency combs leading to sub-radion $f_{\text {ceo }}$ phase excursion. Appl. Phys. B 2007, 86, 219-227.

70. Petit, J.; Goldner, P.; Viana, B. Laser emission with low quantum defect in $\mathrm{Yb}: \mathrm{CaGdAlO}_{4}$. Opt. Lett. 2005, 30, 1345-1347.

71. Lee, J.; Lee, K.; Jang, Y.-S.; Jang, H.; Han, S.; Lee, S.-H.; Kang, K.-I.; Lim, C.-W.; Kim, Y.-J.; Kim, S.-W. Testing of a femtosecond pulse laser in outer space. Sci. Rep. 2014, 4, 5134.

72. Stumpf, M.; Pekarek, S.; Oehler, A.E.H.; Südmeyer, T.; Dudley, J.M.; Keller, U. Self-referencable frequency comb from a $170-\mathrm{fs}, 1.5-\mu \mathrm{m}$ solid-state laser oscillator. Appl. Phys. B 2010, 99, 401-408.

73. Dolgovskiy, V.; Schilt, S.; Bucalovic, N.; Di Domenico, G.; Grop, S.; Dubois, B.; Giordano, V.; Südmeyer, T. Ultra-stable microwave generation with a diode-pumped solid-state laser in the 1.5- $\mu \mathrm{m}$ range. Appl. Phys. B 2014, 116, 593-601.

74. Portuondo-Campa, E.; Paschotta, R.; Lecomte, S. Sub-100 attosecond timing jitter from low-noise passively mode-locked solid-state laser at telecom wavelength, Opt. Lett. 2013, 38, 2650-2653.

75. Portuondo-Campa, E.; Buchs, G.; Kundermann, S.; Lecomte, S. Ultra-low phase noise microwave generation from a diode-pumped solid-state laser. In Proceedings of the 2015 Joint Conference of the IEEE International Frequency Control Symposium \& European Frequency and Time Forum (FCS), Denver, CO, USA, 12-16 April 2015.

76. Portuondo-Campa, E.; Bennès, J.; Kundermann, S.; Lecomte, S. Dual frequency comb interferometer for fast and high resolution optical spectroscopy. In Proceedings of the CLEO/Europe-EQEC 2015, Munich, Germany, 21-25 June 2015.

77. Giesen, A.; Hügel, H.; Voss, A.; Wittig, K.; Brauch, U.; Opower, H. Scalable concept for diode-pumped high-power solid-state lasers. Appl. Phys. B 1994, 58, 365-372. 
78. Saraceno, C.J.; Emaury, F.; Schriber, C.; Hoffmann, M.; Golling, M.; Südmeyer, T.; Keller, U. Ultrafast thin-disk laser with $80 \mu \mathrm{J}$ pulse energy and $242 \mathrm{~W}$ of average power. Opt. Lett. 2014, 39, 9-12.

79. Matos, L.; Mücke, O.D.; Chen, J.; Kärtner, F.X. Carrier-envelope phase dynamics and noise analysis in octave-spanning Ti:Sapphire lasers. Opt. Express 2006, 14, 2497-2511.

(C) 2015 by the authors; licensee MDPI, Basel, Switzerland. This article is an open access article distributed under the terms and conditions of the Creative Commons Attribution license (http://creativecommons.org/licenses/by/4.0/). 